\title{
Remapping in Cerebral and Cerebellar Cortices Is Not Restricted by Somatotopy
}

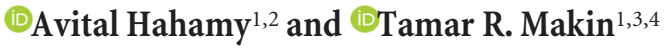 \\ ${ }^{1}$ The Wellcome Centre for Human Neuroimaging, Queen Square Institute of Neurology, University College London, London WC1N 3AR, United Kingdom, \\ ${ }^{2}$ Department of Neurobiology, Weizmann Institute of Science, Rehovot 7610001, Israel, ${ }^{3}$ Institute of Cognitive Neuroscience, University College London, \\ London WC1N 3AZ, United Kingdom, and ${ }^{4}$ Wellcome Centre for Integrative Neuroimaging, University of Oxford, Oxford OX3 9DU, United Kingdom
}

A fundamental organizing principle in the somatosensory and motor systems is somatotopy, where specific body parts are represented separately and adjacently to other body parts, resulting in a body map. Different terminals of the sensorimotor network show varied somatotopic layouts, in which the relative position, distance, and overlap between body-part representations differ. Since somatotopy is best characterized in the primary somatosensory (S1) and motor (M1) cortices, these terminals have been the main focus of research on somatotopic remapping following loss of sensory input (e.g., arm amputation). Cortical remapping is generally considered to be driven by the layout of the underlying somatotopy, such that neighboring body-part representations tend to activate the deprived brain region. Here, we challenge the assumption that somatotopic layout restricts remapping, by comparing patterns of remapping in humans born without one hand (hereafter, one-handers, $n=26$ ) across multiple terminals of the sensorimotor pathway. We first report that, in the cerebellum of one-handers, the deprived hand region represents multiple body parts. Importantly, the native representations of some of these body parts do not neighbor the deprived hand region. We further replicate our previous findings, showing a similar pattern of remapping in the deprived hand region of the cerebral cortex in one-handers. Finally, we report preliminary results of a similar remapping pattern in the putamen of one-handers. Since these three sensorimotor terminals (cerebellum, cerebrum, putamen) contain different somatotopic layouts, the parallel remapping they undergo demonstrates that the mere spatial layout of body-part representations may not exclusively dictate remapping in the sensorimotor systems.

Key words: fMRI; one-handers; plasticity; sensorimotor; somatotopy; topography

\section{Significance Statement}

When a hand is missing, the brain region that typically processes information from that hand may instead process information from other body parts, a phenomenon termed remapping. It is commonly thought that only body parts whose information is processed in regions neighboring the hand region could "take up" the resources of this now deprived region. Here we demonstrate that information from multiple body parts is processed in the hand regions of both the cerebral cortex and cerebellum. The native brain regions of these body parts have varying levels of overlap with the hand regions of the cerebral cortex and cerebellum, and do not necessarily neighbor the hand regions. We therefore propose that proximity between brain regions does not limit brain remapping.

\section{Introduction}

Somatotopic organization in primary somatosensory and motor cortices is thought to reflect the lateralized and segregated neural

Received Oct. 8, 2018; revised July 16, 2019; accepted Aug. 5, 2019.

Author contributions: A.H. contributed unpublished reagents/analytic tools; A.H. analyzed data; A.H. and T.R.M. wrote the first draft of the paper; A.H. and T.R.M. edited the paper; A.H. and T.R.M. wrote the paper; A.H. and T.R.M. designed research; A.H. and T.R.M. performed research.

A.H. was supported by the European Molecular Biology Organization nonstipendiary Long-Term Fellowship 848-2017, Human Frontier Science Program LT000444/2018, Israeli National Postdoctoral Award Program for Advancing Women in Science, the Israeli Presidential Bursary for outstanding PhD students in brain research, Boehringer Ingelheim Fonds travel grant, and the European Union's Horizon 2020 research and innovation programme (under the Marie Sklodowska-Curie Grant Agreement 789040). T.R.M. was supported by a Sir Henry Dale Fellowship (jointly funded by the Wellcome Trust and the Royal Society Grant 104128/Z/14/Z), and the European Research Council Starting Grant 715022 (EmbodiedTech). We thank Alona Cramer, Jan Scholtz, Fiona van der Heiligenberg, activation patterns associated with sensations from, and movements of, distinct body parts (Penfield and Boldrey, 1937; Penfield and Rasmussen, 1950; Catani, 2017; Roux et al., 2018). Following input and output loss (e.g., arm amputation in adults),

Harriet Dempsey Jones, and Lucilla Cardinali for help in data collection; Victoria Root and Arabella Bouzigues for comments on the manuscript; Opcare for help with participant recruitment; and our participants for taking part in these studies.

The authors declare no competing financial interests.

Correspondence should be addressed to Avital Hahamy at a.hahamy@ucl.ac.uk.

https://doi.org/10.1523/JNEUROSCI.2599-18.2019

Copyright $\odot 2019$ Hahamy and Makin

This is an open-access article distributed under the terms of the Creative Commons Attribution License Creative Commons Attribution 4.0 International, which permits unrestricted use, distribution and reproduction in any medium provided that the original work is properly attributed. 
S1/M1 somatotopies undergo remapping, such that the region previously representing the hand becomes responsive to inputs from other body parts (Flor et al., 1995; Makin et al., 2013b; Chand and Jain, 2015; Raffin et al., 2016). The principles governing such architectural change are thought to derive from the underlying somatotopy: neighboring representations, which share greater cortical overlap (Merzenich et al., 1984; Pons et al., 1991; Merzenich and Jenkins, 1993; Florence et al., 1998) and/or receive stronger inhibition from now absent inputs (Faggin et al., 1997; Margolis et al., 2012) are more likely to activate the deprived cortical region. Subsequently, findings showing increased activation by facial inputs in the deprived-hand region following arm amputation (interpreted as resulting from a presumed proximity between hand and lower-face representations) (Jain et al., 2008; Kaas et al., 2008; MacIver et al., 2008; Foell et al., 2014; Andoh et al., 2018), have been taken as evidence for the role of somatotopy in scaffolding remapping.

$\mathrm{We}$, and others, have recently challenged this view, by demonstrating that remapping may occur between both neighboring and distant body-part representations. For example, movements of the intact hand of amputees show increased activation in the S1/M1 deprived-hand region (hereafter, deprived cerebral hand region) (Bogdanov et al., 2012; Makin et al., 2013b; Philip and Frey, 2014). Similarly, individuals born without hands show increased activation in their deprived cerebral hand regions when moving their feet (Stoeckel et al., 2009; Yu et al., 2014; StriemAmit et al., 2018). This feet-to-hands remapping occurs despite the inherent cortical distance between the native regions of the feet and hands. Finally, in individuals born without one hand (hereafter, one-handers), movements of multiple body parts (residual arm, lips, and feet, but not the intact hand) activate the deprived cerebral hand region (Makin et al., 2013b; Hahamy et al., 2017). As the native foot and lip regions are not immediately neighboring the hand region, we suggested that proximity between body-part representations is not a prerequisite for remapping. Yet, it has recently been argued that in cases of congenital hand loss, remapping is driven by topographic constraints, such that body-part representations that are further from the hand region will show reduced remapping compared with representations that neighbor the hand region (Striem-Amit et al., 2018). Thus, local somatotopy is still considered the main driver of remapping in both congenital and late-onset sensorimotor deprivation.

Here, we address this question by examining remapping in one-handers, measuring the level of activation in the deprived hand region evoked by movements of multiple body parts. Crucially, remapping is examined at multiple sensorimotor terminals with varying somatotopies. Somatotopic organization was previously identified throughout the sensorimotor system, such as in the cerebellum (Manni and Petrosini, 2004), brainstem (Jang et al., 2011), and basal ganglia (Nambu, 2011). Here, we focus on the cerebellum, where somatotopy can be reliably identified using fMRI (Grodd et al., 2001; Buckner et al., 2011; Wiestler et al., 2011; Haak et al., 2018). The cerebellum's somatotopy differs from that of S1/M1. For example, in S1/M1 the hand and arm have separate regions, and the lip and foot regions are equally distant from the hand region (Makin et al., 2015). However, in the cerebellum, the arm and hand regions are overlapping, and the lip region partially overlaps with the hand region (Manni and Petrosini, 2004; Mottolese et al., 2013, 2015). If mere somatotopy drives remapping (Flor et al., 1995; Chand and Jain, 2015; Raffin et al., 2016; Striem-Amit et al., 2018), then these different somatotopies should result in different remapping patterns between the cerebral and cerebellar deprived hand regions. However, if similar patterns of remapping would be observed across these terminals, it is less likely that remapping is solely determined by the local somatotopy (Hahamy et al., 2017). We test these competing hypotheses using several independently-acquired datasets of one-handers and two-handed controls and a meta-analysis approach. Our findings provide robust evidence for similar body-part remapping across the hierarchy of the sensorimotor system. We therefore propose that remapping is not necessarily restricted by the physical proximity between the native and remapped representations, and discuss alternative factors that may underlie this extensive brain plasticity.

\section{Materials and Methods}

To avoid known issues of flexibility in fMRI analyses (Carp, 2012) and to enable replication, we harmonized our methods, including experimental design, preprocessing steps, and statistical analyses across datasets, to compare with our previous publication (Hahamy et al., 2017).

\section{Participants}

This study makes use of three independently-acquired fMRI datasets, each containing data of both one-handers and two-handed controls. Two of these datasets had cerebellar coverage and were therefore used for cerebellar analyses. All three datasets were used for analysis of the cerebral cortex (cerebral cortex findings in the third dataset have been published in Hahamy et al., 2017).

Recruitment was performed in accordance with National Health Service national research ethics service approval (10/H0707/29, first dataset) and with Oxford University's Medical Sciences interdivisional research ethics committee (MS-IDREC-C2-2015-012, second dataset). Informed consent and consent to publish was obtained in accordance with ethical standards set out by the Declaration of Helsinki.

The first dataset (hereafter, Dataset1) contained the same population recruited for a previous study, using the same scanning procedures and exclusion criteria as described previously (Hahamy et al., 2015b). Twenty-five healthy controls ( 15 females, age $=41.12 \pm 12.86$ years, 8 left-hand dominant) and 14 individuals with a congenital unilateral upper limb deficit (one-handers, 9 females, age $=36.64 \pm 12.02$ years, 4 with absent right hand) were recruited for the study. The proportion of one-handers with a missing right hand $(n=4)$ and controls who are left-hand dominant $(n=8)$ were $\operatorname{similar}\left(\chi_{(1)}^{2}=0.18, p=0.67\right)$.

The second dataset (hereafter, Dataset2) was acquired as part of a larger study (the full study protocol is currently under preparation and will be made available via Open Science Framework). These data included the scanning of 12 healthy controls ( 5 females, age $=45.33 \pm$ 14.85 years, 5 left-hand dominant $)$ and 14 one-handers ( 7 females, age $=$ $45.25 \pm 11.38$ years, 6 with absent right hand) (for demographic details, see Table 1$)$. The proportions of one-handers with a missing right hand $(n=6)$ and controls who are left-hand dominant $(n=5)$ were similar $\left(\chi_{(1)}^{2}=0.05, p=0.82\right)$. Four one-handers participated in both studies, with data acquired $\sim 5$ years apart.

Full demographic description and acquisition-related information regarding the third dataset are available (Hahamy et al., 2017).

\section{Experimental design}

Scanning protocol for all datasets included multiple scans (see protocol in https://osf.io/4vcmx/). Only an anatomical T1 scan and a task scan for body-part functional localization were used and analyzed here; these scanning procedures were described previously (Makin et al., 2013b; Hahamy et al., 2015b).

The sensorimotor task in all datasets followed the same procedure: Participants were visually instructed to move each of their hands (finger flexion/extension), arms (elbow flexion/extension), feet (bilateral toe movements), or lips, as paced by a visual cue. None of the one-handers experienced phantom sensations. Therefore, in conditions concerning missing hand movements (and elbow movements for 1 participant with an above-elbow deficiency), participants were instructed to imagine moving their missing limb. This condition was only included to match the experimental design across groups and was not used for main analy- 


\begin{tabular}{|c|c|c|c|c|c|}
\hline $\begin{array}{l}\text { Participant } \\
\text { no. }\end{array}$ & $\begin{array}{l}\text { Age, } \\
\text { yr }\end{array}$ & Gender & $\begin{array}{l}\text { Level of limb } \\
\text { deficiency }\end{array}$ & $\begin{array}{l}\text { Affected } \\
\text { limb }\end{array}$ & $\begin{array}{l}\text { Participated in } \\
\text { both studies }\end{array}$ \\
\hline 1 & 39 & Male & Below elbow & Left & Yes \\
\hline 2 & 35 & Male & Below elbow & Right & No \\
\hline 3 & 29 & Female & Below elbow & Left & No \\
\hline 4 & 58 & Male & Below elbow & Left & Yes \\
\hline 5 & 37 & Female & Below elbow & Right & Yes \\
\hline 6 & 52 & Female & Below elbow & Left & No \\
\hline 7 & 32 & Male & Below elbow & Left & No \\
\hline 8 & 61 & Male & Below elbow & Left & No \\
\hline 9 & 42 & Female & Below elbow & Left & No \\
\hline 10 & 53 & Female & Below elbow & Right & Yes \\
\hline 11 & 44 & Male & Below elbow & Left & No \\
\hline 12 & 35 & Male & Below elbow & Right & No \\
\hline 13 & 51 & Female & Below elbow & Left & No \\
\hline 14 & 63 & Female & Below elbow & Right & No \\
\hline
\end{tabular}

${ }^{a}$ Full details of the participants of Dataset1 are available in Hahamy et al. (2015b).

sis. The protocol consisted of alternating $12 \mathrm{~s}$ periods of movement and rest. Each of the six conditions was repeated four times in a semicounterbalanced order. Participants were trained before the scan on the degree and form of the movements. To confirm that appropriate movements were made at the instructed times, task performance was visually monitored online, and video recordings were made in a subset of the scans for further offline evaluation.

\section{MRI data acquisition}

The MRI measurements of Dataset1 were obtained using a 3T Verio scanner (Siemens) with a 32-channel head coil. Anatomical data were acquired using a T1-weighted MPRAGE with the following parameters: TR $2040 \mathrm{~ms}$, TE $4.7 \mathrm{~ms}$, flip angle $8^{\circ}$, voxel size $1 \mathrm{~mm}$ isotropic resolution. Functional data based on the BOLD signal were acquired using a multiple gradient echo-planar $\mathrm{T} 2{ }^{*}$-weighted pulse sequence, with the following parameters: TR $2000 \mathrm{~ms}$, TE $30 \mathrm{~ms}$, flip angle $90^{\circ}$, imaging matrix $64 \times$ 64, FOV $192 \mathrm{~mm}$ axial slices. A total of 46 slices with slice thickness of 3 $\mathrm{mm}$ and no gap were oriented in the oblique axial plane, covering the whole cortex, with partial coverage of the cerebellum.

MRI images of Dataset2 were acquired using a 3T MAGNETON Prisma MRI scanner (Siemens) with a 32-channel head coil. Anatomical images were acquired using a T1-weighted sequence with the following parameters: TR $1900 \mathrm{~ms}$, TE $3.97 \mathrm{~ms}$, flip angle $8^{\circ}$, voxel size $1 \mathrm{~mm}$ isotropic resolution. Functional images were collected using a multiband $\mathrm{T} 2^{*}$-weighted pulse sequence with a between-slice acceleration factor of 4 and no in-slice acceleration. This allowed acquiring data with increased spatial (2 mm isotropic) and temporal (TR $1500 \mathrm{~ms}$ ) resolution, covering the entire brain. The following acquisition parameters were used: TE $32.40 \mathrm{~ms}$, flip angle $75^{\circ}, 72$ transversal slices. Field maps were acquired for field unwarping.

\section{Preprocessing of functional data}

All imaging data were processed using FSL 5.1 (www.fmrib.ox.ac.uk/fsl). Data collected for individuals with absent right limbs were mirror reversed across the mid-sagittal plane before all analyses, so that the hemisphere corresponding to the missing hand was consistently aligned across participants. Data collected for left-hand dominant controls were also flipped, to account for potential biases stemming from this procedure. The proportion of flipped data did not differ between experimental groups in either dataset $\left(\chi_{(1)}^{2}=0.18, p=0.67\right.$ for Dataset $1 ; \chi_{(1)}^{2}=0.05$, $p=0.82$ for Dataset 2 ), and this flipping procedure has been validated using multiple approaches (see Hahamy et al., 2017).

Functional data were analyzed using FMRIB's expert analysis tool (FEAT, version 5.98). The following prestatistics processing was applied to each individual task-based run: motion correction using FMRIB's Linear Image Registration Tool (Jenkinson et al., 2002); brain-extraction using BET (Smith, 2002); mean-based intensity normalization; high pass temporal filtering of $100 \mathrm{~s}$; and spatial smoothing using a Gaussian
Table 2. Number of voxels $\left(2 \mathrm{~mm}^{3}\right)$ and center-of-gravity coordinates of ROls

\begin{tabular}{llr}
\hline & No. of voxels & \multicolumn{1}{c}{ Coordinates } \\
\hline Intact/dominant hand (cerebellum, Dataset1) & 100 & $21,-51,-21$ \\
Deprived/nondominant hand (cerebellum, Dataset1) & 100 & $-21,-52,-21$ \\
Intact/dominant hand (cerebellum, Dataset2) & 100 & $21,-51,-22$ \\
Deprived/nondominant hand (cerebellum, Dataset2) & 100 & $-20,-51,-24$ \\
Intact/dominant hand (cerebrum) & 388 & $38,-22,56$ \\
Deprived/nondominant hand (cerebrum) & 388 & $-38,-22,56$ \\
Intact/dominant putamen & 286 & $26,4,0$ \\
Deprived/nondominant putamen & 321 & $-26,4,0$ \\
\hline
\end{tabular}

kernel of FWHM $4 \mathrm{~mm}$. Time course statistical analysis was performed using FILM (FMRIB's Improved Linear Model) with local autocorrelation correction. Functional data were aligned to structural images (within-subject) initially using linear registration (FMRIB's Linear Image Registration Tool, FLIRT), then optimized using Boundary-Based Registration (Greve and Fischl, 2009). Structural images were transformed to standard MNI space using a nonlinear registration tool (FNIRT), and the resulting warp fields were applied to the functional statistical summary images.

\section{Statistical analyses}

Meta-analysis approach. The current study makes use of three separate datasets, acquired across several years and using different magnets and scanning parameters. Two of these datasets included coverage of the cerebellum and were therefore used for cerebellar analysis, and all three datasets were used for analysis of the cerebral cortex. Multiple datasets can, in principle, be collapsed for analysis purposes, benefiting from statistical power to identify weak effects that may not be noticeable in each separate dataset (Friston, 2012). However, as the current study is guided by an a priori hypothesis that is also spatially focal (remapping in the deprived hand region of one-handers), it calls for more stringent inference methods, rather than for exploratory ones that benefit from enhanced power. We therefore opted to analyze each dataset separately and combine results using a meta-analysis approach (Hahamy et al., 2015a). Differences across datasets are naturally expected, given the inherent variability between datasets (different scanners/scanning protocols/participants) as well as various noise factors that influence any fMRI measurement. However, while interdataset variability could be attributed to both noise and experiment-related phenomena, consistent effects across datasets can only be attributed to the latter. Thus, the use of meta-analysis allowed us to test the inherent reproducibility of findings across datasets, and hence make more valid inferences (Ioannidis et al., 2014; Picciotto, 2018).

Our analysis pipeline for all ROI-based analyses reported below included non-parametric permutation tests performed within each separate dataset. Permutation tests are statistically stringent as they make no assumptions regarding the finite sample distribution of the data, but rather derive it given the data observed (Holmes et al., 1996; Nichols and Holmes, 2002), and are also less sensitive to outlier effects (Masyn et al., 2013), thus contributing to the robustness of findings. The datasetspecific $p$ values resulting from each of the below-described permutation tests were later combined across datasets and meta-analyzed using Fisher's method (Fisher, 1925, 1948) to test the reproducibility of results across datasets. To establish the robustness of the reported effects, $p$ values were additionally tested using Stouffer's test (Stouffer et al., 1949) and the weighted $z$ test (weights set to the square root of each sample size) (Liptak, 1958). To correct for multiple hypotheses testing across the three experimental conditions of interest (movements of the residual arm, lips, and feet), the $\alpha$ level was adjusted to 0.017 based on the highly conservative Bonferroni correction.

Whole-brain analysis. To evaluate whether movements of different body parts differentially activate the brains of one-handers compared with controls, activations evoked by movements of these different body parts were compared between the experimental groups. Movements of the lips and feet were directly compared between groups, intact hand movements in the one-handed group were compared with dominant hand movements in controls, and residual arm movements in the one- 


\section{One-handers $>$ Controls Dataset 1 Dataset 2}

\section{Deprived/ Nondominant hemisphere Dominant hemisphere}

\begin{abstract}
Deprived/ Nondominant hemisphere

Intact/
\end{abstract}

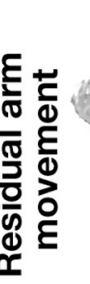

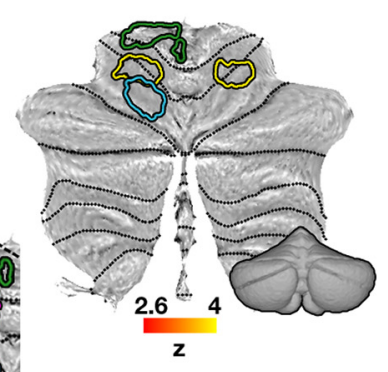
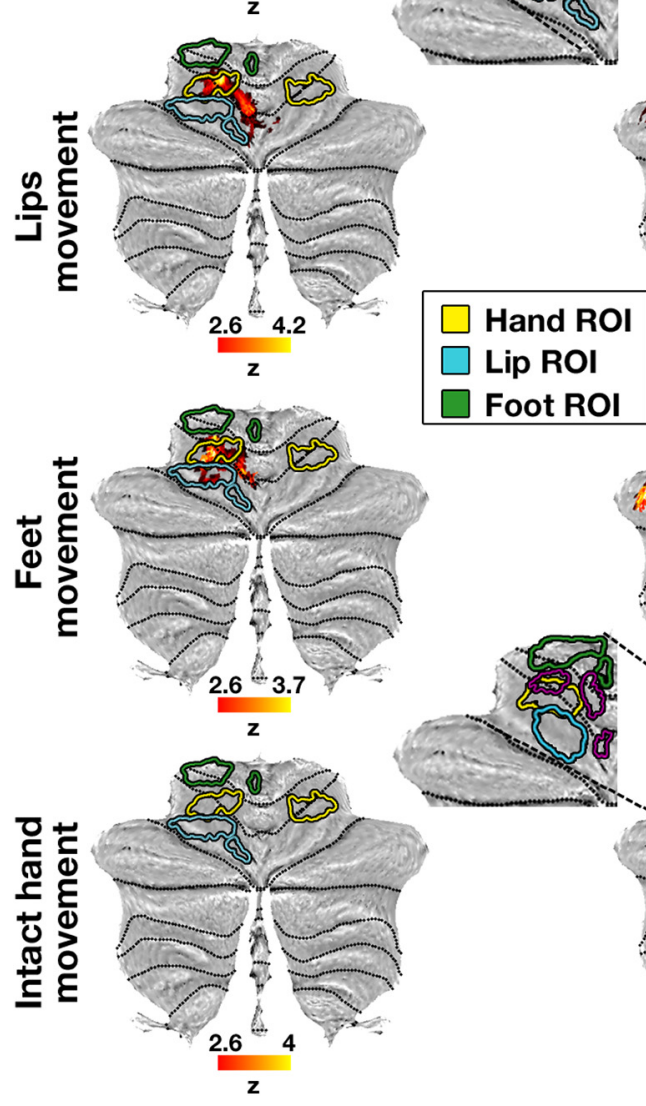

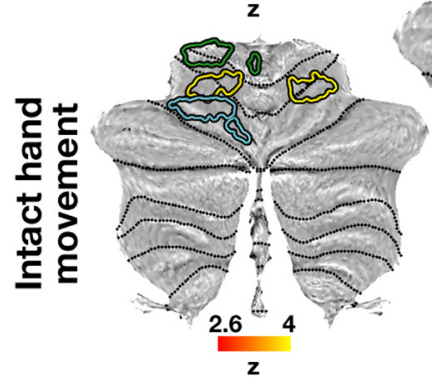

Figure 1. Representation of multiple body parts in the deprived cerebellar hand region of one-handers: between-group contrast maps. Left/Right, Between-group contrast maps of Dataset1/Dataset2, respectively, during residual/nondominant arm (onehanders/controls), lips, feet, and intact/dominant hand movements, projected onto a flat surface of the cerebellum (top right, example of an inflated surface). In the lips and feet conditions (but not in the residual arm or intact hand conditions), one-handers showed increased activation compared with controls, centered on the deprived cerebellar hand region. Yellow/blue/green contours represent the hand/lip/foot ROIs, respectively. Middle, Insets, Independent ROIs used in each of the datasets, defined based on the activations of the other dataset to ensure statistical independence. Purple contours represent the residual arm region. Intact/dominant hemisphere, ipsilateral to the intact/dominant hand; deprived/nondominant hemisphere, ipsilateral to missing/ nondominant hand. All maps were cluster-based corrected for multiple comparisons across the entire brain. Results of residual arm movements in a subset of participants from Dataset1 were previously reported (Makin et al., 2013b).

cluded in the GLM as nuisance regressors. Our main comparisons contrasted intact/dominant hand, residual/nondominant arm, lips, and feet conditions against a baseline (rest) condition.

Second-level analysis of statistical maps was performed using FMRIB's Local Analysis of Mixed Effects (FLAME). The cross-subject GLM included planned comparisons between the two groups. $z$ (Gaussianized $t / F$ ) statistic images were thresholded using clusters determined by $z>2.6(p<0.01)$, and a family-wise error-corrected cluster significance threshold of $p<0.01$ was applied to the suprathreshold clusters. This whole-brain analysis tests the specificity of plasticity to the deprived hand region of one-handers; hence, a lenient statistical threshold ( $p<0.05)$ is typically used in such procedures (Makin et al., 2013b; Hahamy et al., 2017). Nevertheless, as we test several wholebrain comparisons (residual arm, lips, and feet conditions), we chose a more strict threshold of 0.01 across our tests to correct for any $\alpha$ inflation. The nature of the sensorimotor task, in combination with the spatial acquisition resolution, the smoothing and coregistration steps, precludes us from reliably separating sensory and motor subregions. As such, all results are regarded as "sensorimotor."

For visualization purposes only, conditionspecific within-group maps were created for both the cerebellum and cerebral cortex, using the same statistical procedures reported above. These maps were merely aimed at visualizing the sources of the reported group differences, and hence were presented at varying thresholds that best capture the effects observed in the direct statistical comparisons between groups. Specifically, all maps were thresholded at $p<$ 0.01 , except for the cerebral maps of the arm condition, which were thresholded at $p<$ 0.0006 . Since arm movements massively activate the hand region, the choice of a more stringent threshold for these maps enabled a better visualization of the group differences in overlap between peak activity and the deprived hand region. Using the same rationale, maps were presented before correction for multiple comparison to best visualize group differences.

For presentation purposes, statistical parametric activation maps of the cerebellum were projected onto a flat cerebellar surface using SUIT (Diedrichsen and Zotow, 2015), and parametric activations in the cerebral cortex were projected onto an inflated cortical surface of a representative participant using the Connectome Workbench.

Cerebellar regions of interest (ROI) definition. To ascertain that the observed increased cerebellar activation in one-handers (observed across the two datasets with cerebellar coverage) falls within the hand region, and to measure its extent, single-subject activation values

handed group were compared with nondominant arm movements in controls. All statistical analyses were designed to follow the procedures described in our original report (Hahamy et al., 2017). Statistical analyses were conducted using FSL and in-house MATLAB code. To compute task-based statistical parametric maps, we applied a voxel-based GLM, as implemented in FEAT, using a double-gamma hemodynamic response function and its temporal derivative convolved with the experimental model. The six motion parameters and their derivatives were also in- were extracted from independently defined hand-region ROIs and compared between experimental groups. Activations in the control group of each dataset were used to define ROIs for the second dataset (thus keeping the ROI definition independent of the tested data). Thus, to define the cerebellar hand regions of the dominant/intact hemisphere (ipsilateral to the dominant/intact hand) and nondominant/deprived hemisphere (contralateral to the dominant/intact hand), the 100 cerebellar 
voxels of highest activation evoked by either dominant or nondominant hand movements in the control group of one dataset were used as ROIs in the second dataset, and vice versa (Table 2; see Figs. 1, 2). Percent signal change activation values from the individual statistical parametric maps were extracted for the intact and deprived hand ROIs for each participant in the residual/nondominant arm, lips, feet, and intact hand conditions. Since the functional data of one control participant in Dataset1 did not cover the cerebellum, data from this individual were excluded from the cerebellar ROI analysis. The same method was used to define the cerebellar regions of the lips and feet for visualization purposes.

Statistical analysis of cerebellar ROIs. To non-parametrically assess each planned group-contrast (experimental conditions involving movements of different body parts), permutation tests were used within each dataset separately (Holmes et al., 1996; Nichols and Holmes, 2002). In each experimental condition separately, the test statistic was set as the difference between mean group activations in a certain ROI. Next, participants' labels (one-handers or controls) were permuted under the null hypothesis of no group differences in the levels of ROI activation under each experimental condition. Thus, two random experimental groups were created for each condition, and the difference between the groups' mean activation in a given ROI was calculated. This procedure was repeated 10,000 times, creating 10,000 random differences that constructed the null distribution. For each experimental condition, the position of the true (unshuffled) group difference relative to the null distribution was used to obtain a two-sided $p$ value. Using the same pipeline, under the null hypothesis of no two-way interactions between groups and hemispheres (ipsilateral and contralateral to the missing/ nondominant hand), both participants' labels and within-participant hemisphere labels were permuted in each dataset and experimental condition separately. The differences between hemisphere scores were calculated per participant, averaged across participants of the same experimental group, and mean group differences were derived. The position of the true (unshuffled) group difference relative to the null distribution (resulting from 10,000 such iterations) in each experimental condition was used to derive a two-sided $p$ value. Because of the somatotopic arrangement within the sensorimotor terminals, in which the hand and arm representations overlap, movements of the hand and arm evoke much higher activation in the hand region compared with movements of the lips and feet in the typical brain. These known differences in activation levels in the hand region preclude us from running a formal direct comparison across body parts, such as a three-way ANOVA.

To comparatively examine the level of remapping of the foot and lip representations (which do not natively overlap with the hand representation), the activations evoked by lips and feet movements in the deprived cerebellar hand region of one-handers were directly compared using a permutation test, following the same procedure previously detailed (test statistic set to the difference between activations evoked by feet and lip movements). Furthermore, a three-way interaction with factors group (controls/one-handers), hemisphere (intact/deprived), and body part (lips/feet) was calculated.

Assessing remapping in the cerebral cortex. The overlap of participants between Dataset1 and Dataset2 is relatively small (4 of 24 participants), which allowed us to perform the above-described cross-dataset replication analyses for the cerebellum. However, we also aimed to test the reproducibility of our previously reported findings of remapping in the cerebral cortex (Hahamy et al., 2017) using the two current datasets and a previously published dataset, and these contained a larger overlap of participants. Dataset 2 included only 5 participants who also participated in the Hahamy et al. (2017) study (with data acquired $\sim 2$ years apart). However, Dataset1 and the data used by Hahamy et al. (2017) greatly overlapped (12 of 14 participants, with data acquired $\sim 3$ years apart). Hence, cerebral-related results obtained from Dataset 1 should be taken as a measure of a within-group replication over time with regards to Hahamy et al. (2017), rather than as a between-group replication over participants.

Analyses performed on cerebral cortex activations were identical to those described for the cerebellar activations, except for the following differences: (1) For cerebral hand ROIs (in both hemispheres), we used independent ROIs, previously defined based on the original sample of
Table 3. Between-group contrast statistics of activation in the hand regions ${ }^{a}$

\begin{tabular}{llllll}
\hline Brain region & Dataset & & Residual arm & Lips & Feet \\
\hline Cerebellum & 1 & No. of voxels & - & 329 & 246 \\
& & $Z_{\text {max }}$ & - & 5 & 3.94 \\
& & Coordinates & - & $-13,-55,-17$ & $-21,-58,-16$ \\
& 2 & No. of voxels & - & 594 & 623 \\
& & $Z_{\text {max }}$ & - & 4.45 & 4.03 \\
Cerebrum & 1 & Coordinates & - & $-17,-59,-21$ & $-21,-57,-22$ \\
& & No. of voxels & 244 & 439 & 268 \\
& & $Z_{\max }$ & 3.82 & 4.5 & 4.11 \\
& 2 & Coordinates & $46,-20,58$ & $45,-17,54$ & $44,-20,58$ \\
& & No. of voxels & 422 & 411 & 450 \\
& & $Z_{\text {max }}$ & 5.79 & 4.24 & 4.14 \\
& & Coordinates & $49,-20,56$ & $48,-16,54$ & $52,-22,50$ \\
\hline
\end{tabular}

${ }^{a}$ The number of voxels, peak intensity $\left(z_{\max }\right)$, and coordinates of the center of gravity of hand-region activations in the cerebellum and cerebrum are presented for each dataset (rows) and task-condition (columns). Coordinates are based on the MNI 152 brain template.

one-handers and controls in our previous study (Hahamy et al., 2017) (see Table 2; these ROIs will be made freely available via open science framework), to standardize the analysis across the three datasets (the two current ones and the previously published one). ROIs for lips and feet were also adopted from the same previous work to visualize the S1/M1 somatotopy. We were unable to reliably separate between the two cerebral foot regions for two reasons. First, our experimental task comprised of simultaneous movements of the two feet. Second, the resulting activation in the two foot regions occupied the medial surface of the cerebral cortex, mixing signals from the two hemispheres due to acquisition, preprocessing, and coregistration parameters. For this reason, a bilateral ROI was defined for the feet. (2) Since the cerebral-focused ROI analyses were guided by a predefined hypothesis (over-representation of the residual arm, lips, and feet in the deprived hand region of one-handers) based on our previous study, one-tailed statistical tests within each of Dataset 1 and Dataset2 were used. Since our previous study did not find a significant interaction between groups and hemispheres for the feet condition, the tests of this effect in the two current datasets were performed in a two-tailed form.

For each experimental condition, sets of 3 dataset-specific $p$ values (resulting from each of the two current datasets, as well as the previously reported $p$ values of the original dataset) were combined and tested using the same methods described for the cerebellum analyses, including correction for multiple comparisons.

Comparing remapping in the cerebellar and cerebral cortices. To compare the relative levels of remapping of the lips and feet into the cerebrum and cerebellum hand regions, the ratio between lips-evoked and feetevoked activation was calculated in the deprived hand region of the cerebrum and cerebellum for each one-hander. The cerebrum remapping ratio was then divided by the cerebellum remapping ratio, and the resulting ratios were averaged across participants of the same dataset to form the test's statistic. A ratio that significantly deviates from 1 would suggest a difference in relative remapping between the cerebrum and cerebellum. Under the null hypothesis of no difference between cerebral and cerebellar remapping ratios, the cerebral and cerebellar remapping ratios were shuffled within participants and then averaged across participants, a procedure that was repeated 10,000 times to create the null distribution. The position of the true (unshuffled) test statistic within this distribution was then used to obtain a two-sided $p$ value. Finally, the resulting datasetspecific $p$ values were tested using Fisher's method to assess the consistency of effects across the two datasets.

Assessing remapping in the putamen. Remapping in the putamen was studied using a more exploratory approach compared with the approach used for the study of the cerebellar and cerebral cortices. The deprived and intact putamen ROIs were defined based on the Harvard-Oxford probabilistic atlas, at a probabilistic threshold of 90 (Table 2). Activations evoked by body part movements in these ROIs were compared between the experimental groups of Dataset2 alone (which had better spatial resolution, allowing the study of this smaller subcortical structure), using two-sided permutation tests, as described for the cerebellar ROI analyses. 
Table 4. Between-group contrast statistics of increased activation in one-handers compared with controls outside the hand regions ${ }^{a}$

\begin{tabular}{|c|c|c|c|c|}
\hline Dataset & Condition & Region & Region & Region \\
\hline 1 & Lips & $\begin{array}{l}\text { Supracalcarine cortex, } 735 \text { voxels, } \\
\qquad z_{\max }=3.75(2,-79,13)\end{array}$ & $\begin{array}{l}\text { Deprived/nondominant occipital pole, } 342 \text { voxels, } \\
\qquad Z_{\max }=4.01(20,-91,23)\end{array}$ & $\begin{array}{l}\text { Intact/dominant parahippocampal gyrus, } 217 \text { voxels, } \\
\qquad Z_{\max }=3.9(-26,-10,-23)\end{array}$ \\
\hline 2 & Lips & $\begin{array}{l}\text { Deprived/nondominant putamen, } 288 \text { voxels, } \\
\qquad z_{\max }=3.7(26,-4,-1)\end{array}$ & $\begin{array}{l}\text { Intact/dominant parahippocampal gyrus, } 187 \text { voxels, } \\
\qquad Z_{\max }=3.83(-30,-17,-28)\end{array}$ & $\begin{array}{l}\text { Deprived/nondominant cerebellar lobule VIIlb, } 167 \text { voxels, } \\
\qquad z_{\max }=4(-7,-78,-48)\end{array}$ \\
\hline & Feet & $\begin{array}{l}\text { Intact/dominant frontal pole, } 221 \text { voxels, } \\
\qquad z_{\max }=4.18(-27,57,0)\end{array}$ & $\begin{array}{l}\text { Deprived/nondominant frontal pole, } 177 \text { voxels, } \\
z_{\max }=3.65(29,49,7)\end{array}$ & - \\
\hline
\end{tabular}

${ }^{a}$ The number of voxels, peak intensity $\left(z_{\max }\right)$, and coordinates of the center of gravity of significant activation clusters are presented for each dataset and task condition. Coordinates are based on the MNI 152 brain template.

\section{Dataset 1}

\section{Controls}

\begin{tabular}{|c|c|c|}
\hline $\begin{array}{l}\text { Deprived/ } \\
\text { Nondominant } \\
\text { hemisphere }\end{array}$ & $\begin{array}{c}\text { Intact/ } \\
\text { Dominant } \\
\text { hemisphere }\end{array}$ & $\begin{array}{l}\text { Deprived/ } \\
\text { Nondominant } \\
\text { hemisphere }\end{array}$ \\
\hline
\end{tabular}
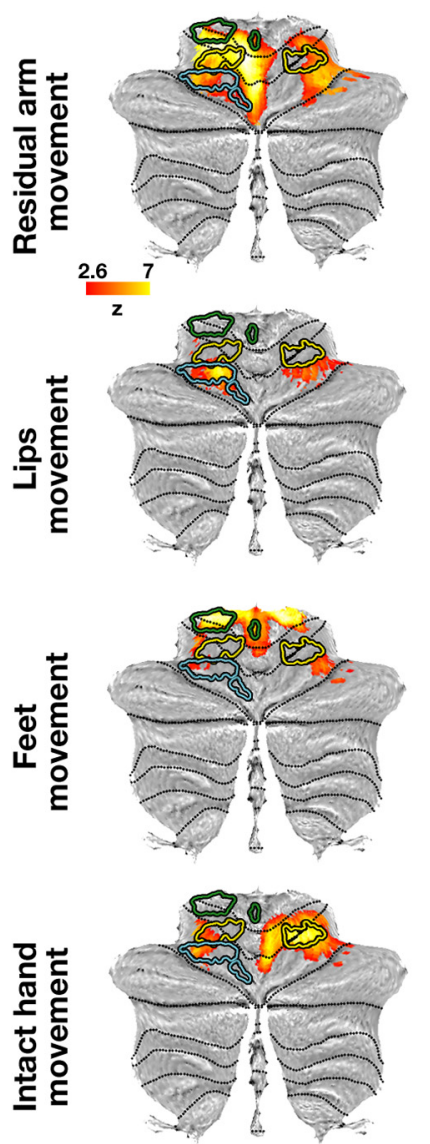
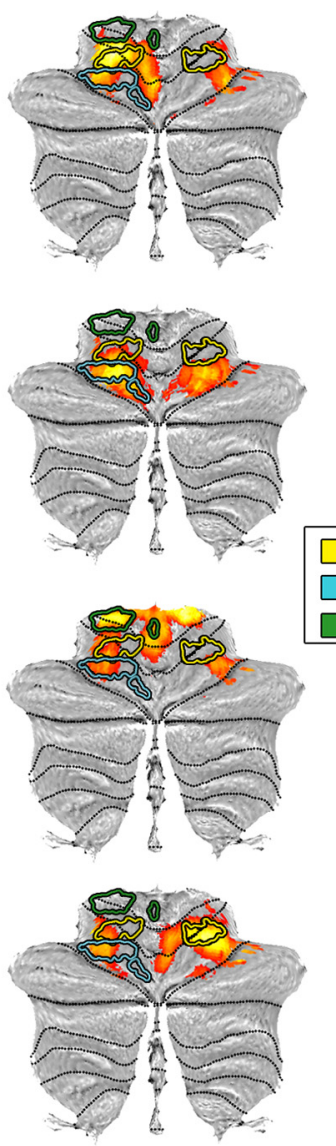

Controls

\section{Dataset 2}

$\begin{array}{cccc}\begin{array}{c}\text { Deprived/ } \\ \text { Nondominant } \\ \text { hemisphere }\end{array} & \begin{array}{c}\text { Intact/ } \\ \text { Dominant } \\ \text { hemisphere }\end{array} & \begin{array}{c}\text { Deprived/ } \\ \text { Nondominant } \\ \text { hemisphere }\end{array} & \begin{array}{c}\text { Intact/ } \\ \text { Dominant } \\ \text { hemisphere }\end{array}\end{array}$
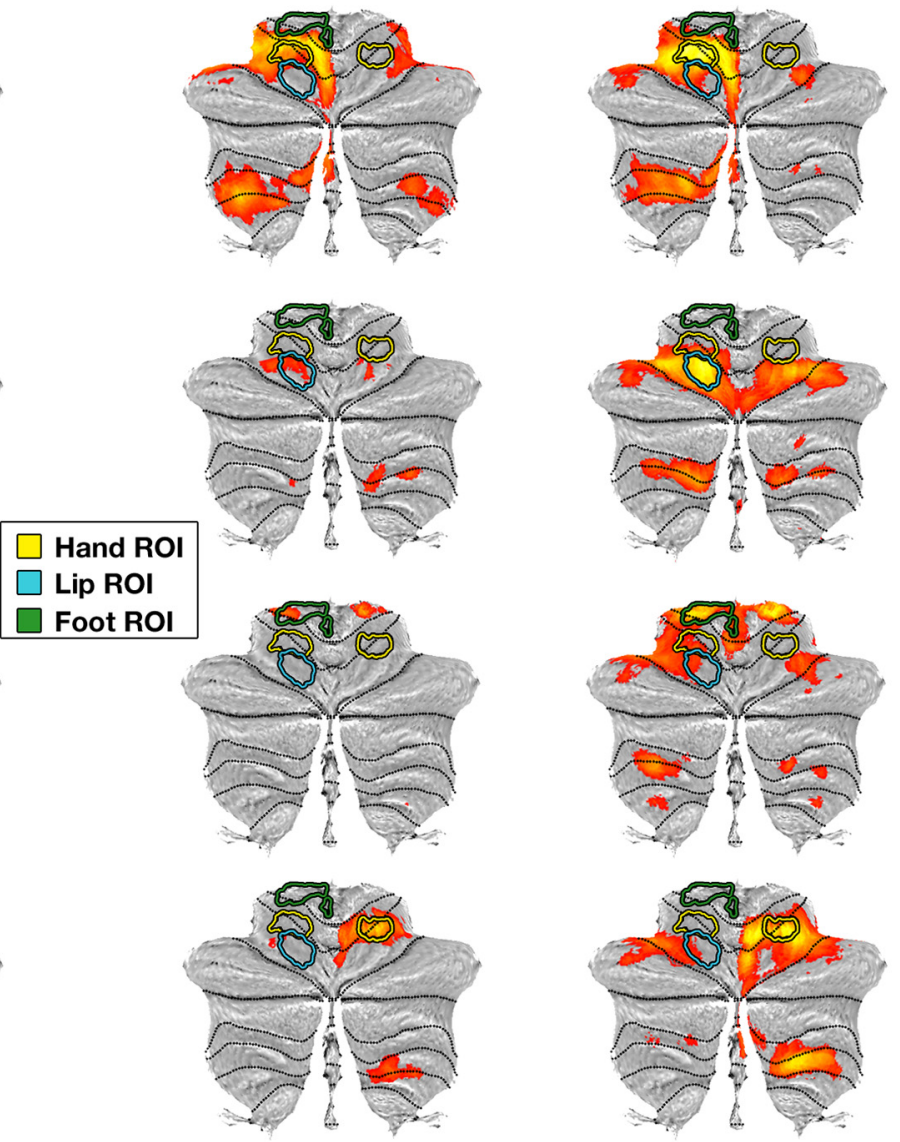

Figure 2. Cerebellar within-group activation maps. Within-group activation maps for each experimental condition versus a resting baseline (rows) are presented for the control and one-handed groups of each separate dataset (columns). All annotations are as detailed in Figure 1. All maps are presented at an uncorrected threshold of $p<0.01$ to visualize the origin of the between-group contrast results presented in Figure 1. The within-group maps of one-handers show overactivation in the cerebellar deprived hand region in the residual arm, lips, and feet conditions, compared with controls.

Confirmatory analysis-spatial layout of body part representations. Finally, we aimed to confirm previously reported results, by demonstrating differences between the native somatotopies of the cerebellum and S1/M1 (see Introduction). To measure the proximity between native body-part representations, the level of overlap between activations evoked by movements of the hand, lips, and feet in the "intact" hemisphere (cerebral hemisphere contralateral to the dominant/intact hand and cerebellar hemisphere ipsilateral to the dominant/intact hand) was measured in all participants using the Dice coefficient (Dice, 1945; Kikkert et al., 2016) as follows:

$$
\frac{2 \times|\mathrm{A} \cap \mathrm{B}|}{|A|+|B|}
$$

Where A and B represent activations evoked by movements of specific body parts (intact hand and lips or intact hand and feet) within a sensorimotor mask. To that end, for each participant, the activation maps of intact hand, lips, and feet conditions were set to a minimal threshold of $z=2$ to allow a relatively wide spread of activation (Kikkert et al., 2016). The few participants who had particularly low spread of activation $(<25$ voxels; representing $2.5 \%$ of the voxels across all analyzed ROIs) in the 
intact hemisphere in either condition, despite the relatively lenient threshold, were excluded from this particular analysis (Dataset1, 3 control participants; Dataset2, 1 one-hander and 1 control participant). In the cerebral cortex, the level of overlapping activations between the hand condition and each of the lips and feet conditions was assessed within a mask of the left precentral gyrus, taken from the Harvard-Oxford probabilistic atlas (this mask was used without setting a threshold, to contain the central sulcus and both the precentral and postcentral gyri; see Fig. $8 A$ ). In the cerebellar cortex, the level of overlapping activations between the hand condition and each of the lips and feet conditions was assessed within a mask of right lobules I-IV, V, and VI, taken from FSL's cerebellar probabilistic atlas. Each of these three cerebellar masks was thresholded at 50 before their unification to restrict the unified mask to the sensorimotor sections of the cerebellar anterior lobe (see Fig. 8A).

For each participant, 4 Dice coefficients were calculated: overlap between intact hand and feet activations, and overlap between intact hand and lips activations, in each of the cerebrum/cerebellum masks separately. We next aimed to verify that the overlap relationship of body part representations differs between the cerebrum and the cerebellum, as previously reported (see Introduction). However, a direct comparison between overlap in representations in the cerebrum versus cerebellum may be confounded by the different spatial scales of these two structures. We therefore targeted a comparison between intrastructure overlap relations, which we will refer to as "neighborhood relationship" of each of the cerebral or cerebellar cortices. This neighborhood relationship was defined as the ratio of lips-hand overlap to feet-hand overlap in each brain structure (cerebrum/cerebellum; see Fig. $8 C$ ). As neighborhood relationships are devised as ratios within each brain structure, they normalize the Dice coefficients and enable a comparison between the cerebrum and cerebellum.

To evaluate whether these neighborhood relationships are different between the cerebrum and cerebellum, a permutation test was used within each dataset. The test's statistic was defined as the crossparticipant mean ratio between cerebellar neighborhood relationship and cerebral neighborhood relationship (a ratio that significantly deviates from 1 would suggest a difference in topographies between the cerebrum and cerebellum). To this end, for each participant, the cerebellar neighborhood relationship was divided by the cerebral neighborhood relationship. Under the null hypothesis of no difference between cerebral and cerebellar neighborhood relationships, the cerebral and cerebellar neighborhood relationships were shuffled within participants and then averaged across participants, a procedure that was repeated 10,000 times to create the null distribution. The position of the true (unshuffled) test statistic within this distribution was then used to obtain a two-sided $p$ value. Finally, the resulting dataset-specific $p$ values were tested using Fisher's method to assess the consistency of affects across the two datasets.

\section{Results}

\section{Cerebellar remapping is not restricted by somatotopy}

To test whether somatotopy restricts remapping in the cerebellum, we assessed remapping of the representations of the residual arm (overlapping the hand region; see Fig. 1, insets), as well as the lips and feet (whose representations have differing levels of overlap with the hand region, see Confirmatory analysis below) in one-handers compared with controls. To this end, we compared results across two independently acquired datasets of onehanders and controls, who underwent an fMRI scan, involving simple movements of the hand, arm, lips, and feet. Whole-brain activations evoked by movements of the residual arm, lips, and feet (body part representations previously shown to remap in the cerebral cortex) (Hahamy et al., 2017), and of the intact hand (whose representation did not show such remapping) (Hahamy et al., 2017) were compared between experimental groups within each dataset. These analyses revealed that movements of the lips and feet, but not movements of the intact hand, excessively acti-
Table 5. Dataset-specific $p$ values per brain region and experimental condition ${ }^{a}$

\begin{tabular}{|c|c|c|c|c|c|c|}
\hline Brain region & Test & Dataset & $\begin{array}{l}\text { Residual } \\
\text { arm }\end{array}$ & Lips & Feet & $\begin{array}{l}\text { Intact } \\
\text { hand }\end{array}$ \\
\hline \multirow[t]{4}{*}{ Cerebellum } & \multirow[t]{2}{*}{ Group contrasts } & 1 & 0.02 & 0.002 & 0.04 & 0.49 \\
\hline & & 2 & 0.02 & 0.004 & 0.001 & 0.38 \\
\hline & \multirow[t]{2}{*}{ Group $\times$ hemisphere interaction } & 1 & 0.04 & 0.009 & 0.11 & 0.46 \\
\hline & & 2 & 0.0008 & 0.007 & $<0.001$ & 0.4 \\
\hline \multirow[t]{6}{*}{ Cerebrum } & \multirow[t]{3}{*}{ Group contrasts } & 1 & $<0.001$ & 0.003 & $<0.001$ & 0.37 \\
\hline & & 2 & 0.03 & $<0.001$ & 0.004 & 0.28 \\
\hline & & 3 & $<0.001$ & $<0.001$ & 0.04 & 0.15 \\
\hline & \multirow[t]{3}{*}{ Group $\times$ hemisphere interaction } & 1 & 0.03 & 0.04 & $<0.001$ & 0.43 \\
\hline & & 2 & 0.05 & 0.02 & 0.15 & 0.16 \\
\hline & & 3 & $<0.001$ & 0.002 & 0.23 & 0.25 \\
\hline
\end{tabular}

${ }^{a}$ Dataset-specific $p$ values (rows) are derived from permutation tests for each experimental condition (columns) for both cerebellar and cerebral hand ROls (top/bottom of table, respectively). Results of Dataset3 were previously reported in Hahamy et al. (2017). Results for the residual arm condition in a subsample of participants from Dataset1 were reported in Makin et al. (2013b).

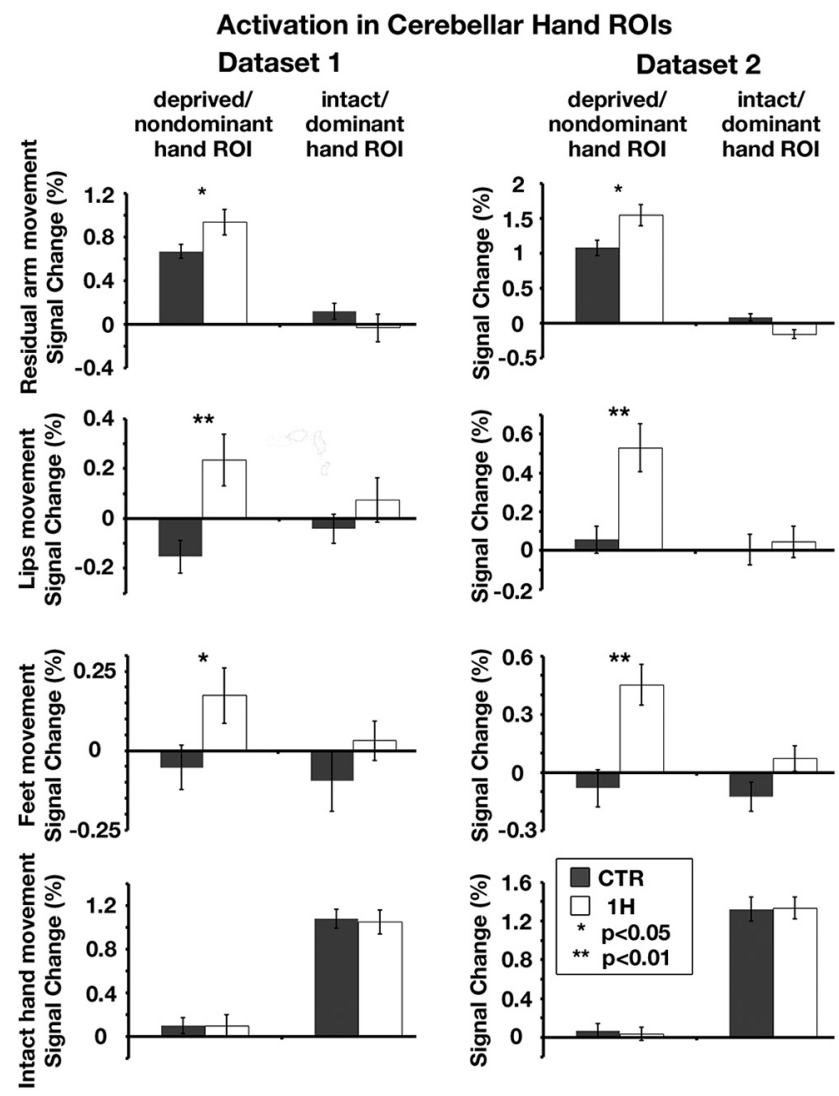

Figure 3. Multiple body parts activate the deprived cerebellar hand region of one-handers: ROI analysis. Left/Right, Activation levels in Dataset1/Dataset2 (respectively) in the bilateral cerebellar hand regions (independently defined for each dataset, ROls depicted in Figs. 1, 2), during residual/nondominant arm (one-handers/controls), lips, feet, and intact/dominant hand movements. Activation levels in the deprived cerebellar hand region of one-handers (white bars) were greater than activations in the nondominant hand region of controls (gray bars) in all but the intact hand condition. $1 \mathrm{H}$, One-handers; (TR, controls; intact/dominant hand ROI, ipsilateral to the intact/dominant hand; deprived/nondominant hand ROI, ipsilateral to missing/nondominant hand. Error bars indicate SEM. Results of residual arm movements in a subset of participants from Dataset1 were previously reported (Makin et al., 2013b). The scales of brain activations ( $y$ axes) are not fixed across experimental conditions, to allow better visualization of the intergroup and interhemispheric differences within each condition.

vated a region in lobules V/VI of the cerebellar hemisphere ipsilateral to the missing hand in one-handers, compared with controls (Fig. 1; Table 3). These activation clusters overlapped with an independently defined ROI of the deprived hand region of the anterior cerebellum (see Materials and Methods; Fig. 1). 


\section{One-handers > Controls Dataset 1 Dataset 2}
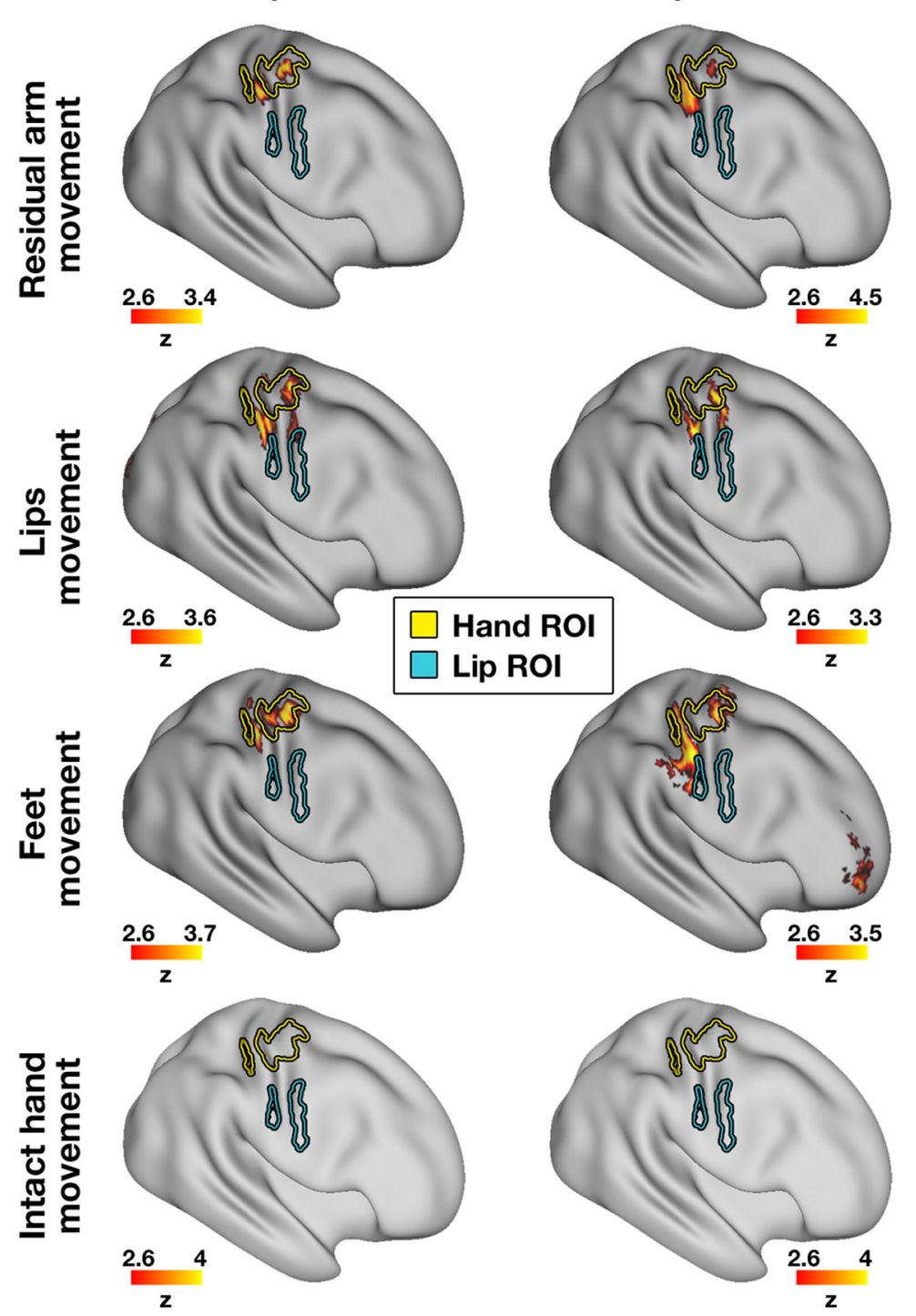

Figure 4. Multiple body parts activate the deprived cerebral hand-region of one-handers: between-group contrast maps. Left/Right, Between-group contrast maps of Dataset1/Dataset2, respectively, during residual/nondominant arm (one-handers/ controls), lips, feet, and intact/dominant hand movements, projected onto an inflated surface of a template brain. In each of the arm, lips, and feet (but not intact hand) conditions, one-handers showed increased activation compared with controls, centered on the deprived cerebral hand region. Yellow/blue contours represent the hand/lip ROls, respectively. Deprived/nondominant hemisphere, contralateral to missing/nondominant hand. All maps were cluster-based corrected for multiple comparisons across the entire brain. Results of residual arm movements in a subset of participants from Dataset1 were previously reported (Makin et al., 2013b).

Unlike our previous findings in the cerebral cortex, the wholebrain between-group contrast did not reveal increased activation in the cerebellar hand region of one-handers during residual arm movements, compared with controls. This could potentially stem from near-complete overlap between the arm and hand representations found in the cerebellum (Mottolese et al., 2013) (Fig. 1, insets). Specifically, if the arm representation natively overlaps with the hand representation, additional remapping between these representations in one-handers may be too subtle to be detected using a whole-brain analysis. Additional clusters showing increased activation in one-handers compared with controls were also found within specific datasets; but unlike the effects in deprived hand region, these clusters were not consistent across all datasets and task conditions (Table 4). The results of the direct between-group contrasts were further visualized using within-group activation maps of each condition versus a rest baseline (Fig. 2).

We next aimed to measure the degree of remapped activations in the deprived cerebellar hand region during movements of different body parts, and assess their consistency across datasets. To this end, within each dataset and movement condition separately, between-group permutation tests were used to compare mean fMRI activation values (percent signal change) obtained from two independent hand ROIs (deprived and intact hand regions of the cerebellar hemispheres' anterior lobe, ROIs depicted in Figs. 1, 2). These ROIs were obtained from the control group of one dataset and tested on the other dataset, and are completely independent of the between-group contrast analysis reported above. Results of these tests were combined across the two datasets (see Materials and Methods). Dataset-specific $p$ values for all experimental conditions are presented in Table 5. As shown in Figure 3, these analyses confirmed increased activation in the deprived cerebellar hand ROI when onehanders moved their lips $\left(\chi_{(4)}^{2}=23.21\right.$, $p<0.001, \alpha=0.017)$ and feet $\left(\chi_{(4)}^{2}=\right.$ 19.91, $p<0.001, \alpha=0.017)$, as well as their residual $\operatorname{arm}\left(\chi_{(4)}^{2}=15.29, p=\right.$ 0.004, $\alpha=0.017$, Bonferroni-corrected, Fisher's method for all tests), compared with controls. Movements of the intact hand (whose representation does not remap in the cerebral cortex) (Hahamy et al., 2017) did not result in increased activation in the deprived cerebellar hand region of one-handers $\left(\chi_{(4)}^{2}=3.33, p=\right.$ 0.51 , Fisher's method). In addition, twoway interactions were consistently revealed between hemispheres and groups (nondominant/residual arm: $\chi_{(4)}^{2}=$ 20.61, $p<0.001$; lips: $\chi_{(4)}^{2}=19.39, p<$ 0.001 ; feet: $\chi_{(4)}^{2}=19.23, p<0.001$, Fisher's method, $\alpha=0.017$, Bonferronicorrected for all tests). These interactions reflect dissociated recruitment of the deprived cerebellar hand region by movements of various body parts in one-handers, in comparison with the intact cerebellar hand region and with the control group (Fig. 3 ). These findings echo the pattern of remapping we previously reported in the cerebral cortex of one-handers, and reflect sensorimotor remapping, which is not limited to the immediate neighbors overlapping with the deprived hand region.

To further evaluate the interplay between remapping and somatotopy, the remapping levels of the lip and foot representations in one-handers were directly compared. If somatotopy 


\section{Dataset 1}

Controls
Intact/ Deprived/
Dominant Nondominant hemisphere hemisphere

\section{One-handers}

\section{Dataset 2}

\section{Controls}

$\begin{array}{cc}\text { Intact/ } & \begin{array}{c}\text { Deprived/ } \\ \text { Dominant } \\ \text { Nondominant } \\ \text { hemisphere }\end{array} \\ \text { hemisphere }\end{array}$

$\begin{array}{cc}\text { Intact/ } & \begin{array}{c}\text { Deprived/ } \\ \text { Dominant } \\ \text { Nondominant } \\ \text { hemisphere }\end{array} \\ \text { hemisphere }\end{array}$

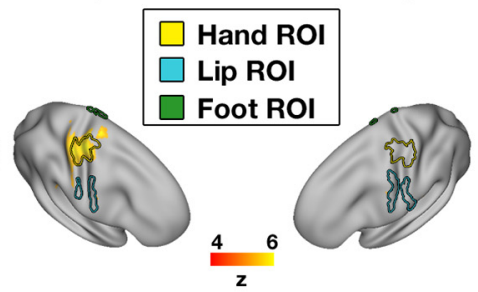

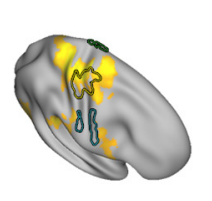
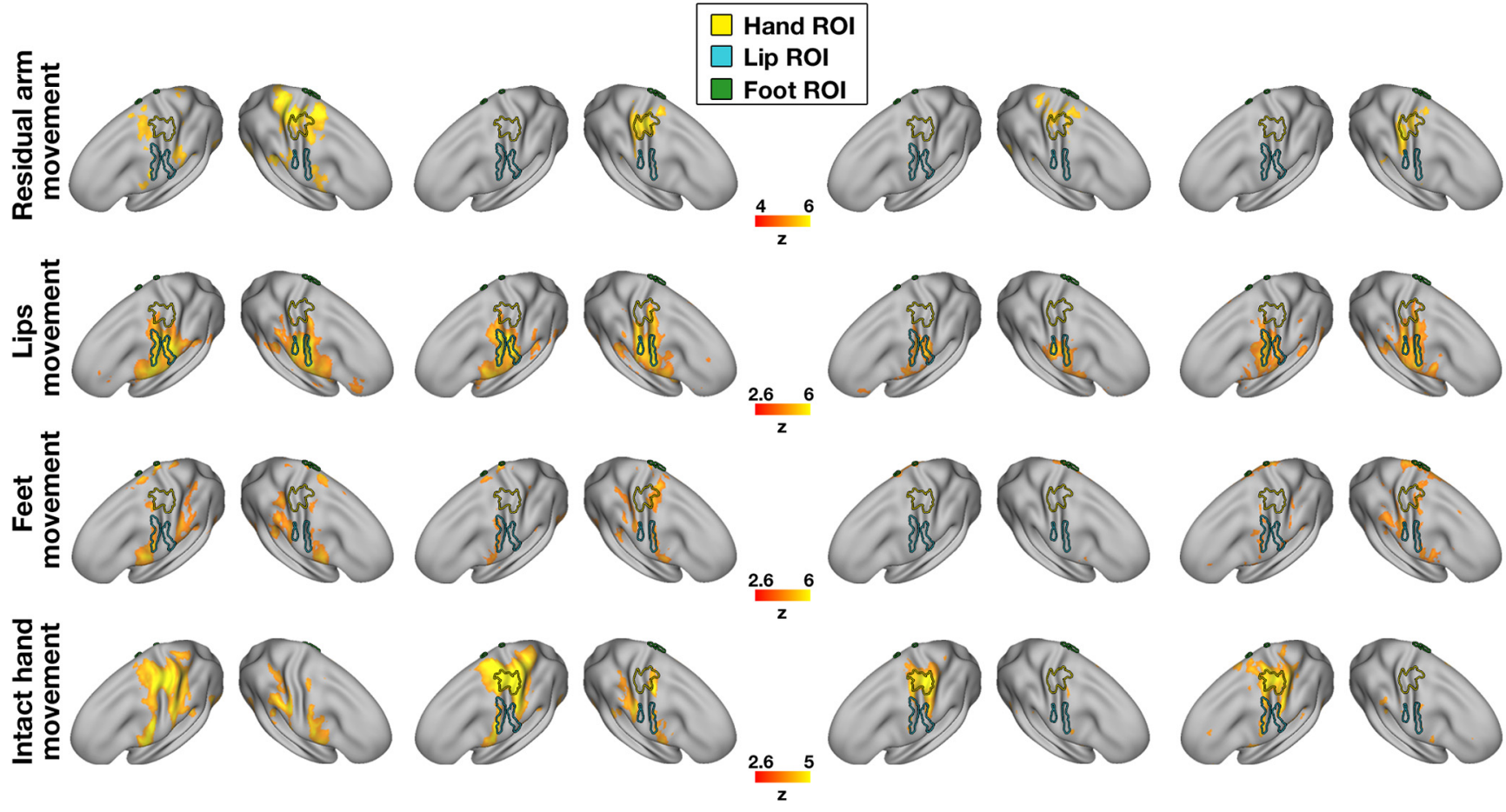

Figure 5. Cerebral within-group activation maps. Within-group activation maps for each experimental condition versus a resting baseline (rows) are presented for the control and one-handed groups of each separate dataset (columns). ROls were defined based on Dataset3, to ensure full replication of our previously published results in this dataset (Hahamy et al., 2017). All annotations are as in Figure 4. All maps are presented as means of visualization of the origin of the between-group contrast results presented in Figure 4, and are therefore presented at varying uncorrected thresholds. The within-group maps of one-handers show overactivation in the cerebral deprived hand region in the residual arm, lips, and feet conditions, compared with controls. Within-group maps of Dataset3 can be found in Hahamy et al. (2017).

drives remapping, the lip representation, which overlaps with the deprived hand representation, should show more remapping compared with the foot representation, which does not overlap with the hand representation (see also Confirmatory analysis below). However, despite different levels of overlap with the cerebellar hand region, no difference was found between lip and foot remapping into this region (Dataset $1, p=0.33$; Dataset $2, p=$ 0.32; permutation tests). Furthermore, a three-way ANOVA with factors group (controls/one-handers), hemisphere (intact/deprived), and body part (lips/feet) revealed no three-way interaction (Dataset1: $F_{(1,36)}=1.86, p=0.18$; Dataset2: $F_{(1,24)}=0.47$, $p=0.5)$. These results do not support the conjecture that somatotopy determines the degree of remapping. However, these null results do not allow a formal interpretation.

Similar pattern of remapping seen in the cerebellar and cerebral deprived hand regions of one-handers

We next used these two datasets to test the reproducibility of our previous findings of remapping in the cerebral cortex of onehanders (our previously reported results were based on Dataset3, and can be found in Hahamy et al., 2017). As shown in Figure 4 (and further visualized in Fig. 5), movements of the residual arm, lips, or feet, but not movements of the intact hand, activated the deprived S1/M1 hand region to a greater extent in one-handers compared with controls, as shown using whole-brain betweengroup contrast maps (see also Table 3). Additional clusters show- ing increased activation in one-handers compared with controls were also found within specific datasets; but unlike the effects in the deprived hand region, these clusters were not consistent across all datasets and task conditions (Table 4). These results were further supported by ROI analyses (ROIs presented in Figs. $4,5)$. To assess reproducibility, the results of the ROI analyses were combined across the two current datasets as well as the dataset used in our previous study (dataset-specific $p$ values for all experimental conditions are presented in Table 5). As depicted in Figure 6 and in Table 6, these tests confirmed increased activation in the deprived cerebral hand ROI when one-handers moved their residual arm, lips, and feet compared with controls. Group $\times$ hemisphere interactions consistently revealed dissociated recruitment of the deprived cerebral hand region (compared with the intact hand region) by movements of various body parts between one-handers and controls (Table 6). Table 7 presents the results of integration across datasets using additional meta-analysis measures (see Materials and Methods) for both the cerebral and cerebellar hand regions.

To evaluate whether different somatotopic layouts would relate to different patterns of body-part remapping, we compared the remapping seen in the deprived cerebellar and deprived cerebral hand regions of one-handers (see Material and Methods). This analysis revealed that, although the cerebellum and cerebrum have different somatotopic layouts (see also Confirmatory 

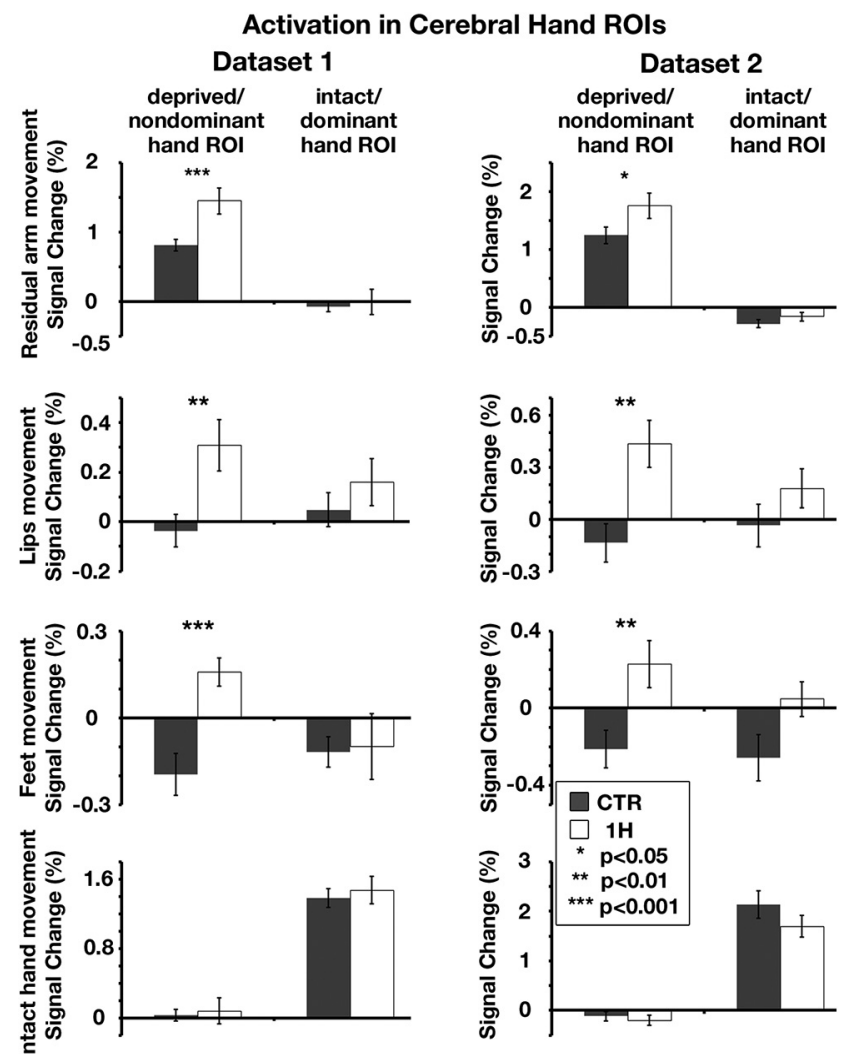

Figure 6. Multiple body parts activate the deprived cerebral hand-region of one-handers: ROI analysis. Left/Right, Activation levels in Dataset1/Dataset2 (respectively) in the bilateral cerebral hand regions (independently defined, ROls depicted in Fig. 5), during residual/nondominant arm (one-handers/controls), lips, feet, and intact/dominant hand movements. Activation levels in the deprived cerebral hand region of one-handers (white bars) were greater than in the nondominant-hand region of controls (gray bars) in all but the intact hand condition. All annotations are as in Figure 3. Results of residual arm movements in a subset of participants from Dataset1 were previously reported (Makin et al., 2013b). The scales of brain activations are not fixed across experimental conditions, to allow a better visualization of the intergroup and interhemispheric differences within each condition.

analysis below), the lip to foot remapping ratios did not differ between these two sensorimotor terminals (Dataset1, $p=0.09$; Dataset2, $p=0.15$; permutation tests; $\chi_{(4)}^{2}=8.6, p=0.072$, Fisher's method). But as noted above, null results should be interpreted with caution.

\section{Remapping in the putamen}

Since the putamen has previously been shown to contain a somatotopic map (Nambu, 2011), and since the basal ganglia has reciprocal connections with both the cerebellum and the primary sensorimotor cortex (Nambu, 2011; Dum et al., 2014; Zeharia et al., 2015), we wished to explore remapping patterns in this terminal. A challenge in studying this area is that its somatotopy is substantially more compact than that of the cerebellum, requiring increased spatial resolution, which was only available in Dataset2. Figure 7 (left) depicts uncorrected between-group contrast maps of Dataset2. Similar to our results in the cerebellum and in S1/M1, these maps demonstrated increased activation in the deprived putamen in the residual arm, lips, and feet conditions, but not in the intact hand condition, in one-handers compared with controls. Only results of the lips condition survived correction for multiple comparisons over the whole brain (Table 4).
ROI analysis provides a more sensitive test for remapping. As depicted in Figure 7 (right), these tests revealed significantly increased activation in the deprived putamen in the residual arm $(p=0.01)$, lip $(p<0.001)$, and feet conditions $(p=0.02$; permutation tests, $\alpha=0.05$ ), but not in the intact hand condition $(p=0.1)$. These effects were accompanied by near-significant group (controls/one-handers) by hemisphere (intact/deprived putamen) interactions (residual arm, $p=0.048$; lip, $p=0.007$; feet, $p=0.057$, permutation tests, $\alpha=0.05$ ), demonstrating the specificity of this effect to the deprived putamen. Unlike in the cerebrum and cerebellum, activation in the putamen tends to be bilateral (Gerardin et al., 2003), hence the non-negligible activation levels in both hemispheres.

\section{Confirmatory analysis: neighborhood relationship between body-part representations differs between the cerebrum and cerebellum}

In both the cerebral and cerebellar cortices, the hand representation resides between the foot and lip representations; however, the level of overlap between these representations was previously reported to differ between the two brain structures (see Introduction). To confirm this difference in overlap between body-part representations (hereafter, neighborhood relationship) in the cerebrum and cerebellum, we studied the overlap in activations evoked by movements of these body part in controls, and onehanders' intact hemisphere (no between-group differences were found in the intact hemisphere).

We confined activations to the sensorimotor parts of the cerebrum and of the cerebellar anterior lobe (Fig. $8 A$ ), and used the Dice coefficient to measure overlap (Dice, 1945; Kikkert et al., 2016) (see Materials and Methods). As demonstrated in the intact/dominant hemispheres of controls and one-handers in Figure $8 B$, some degree of overlap was indeed observed between the peripheral aspects of the hand region and the activations evoked by lips and feet movements in both the cerebrum and cerebellum. This level of overlap was evaluated using permutation tests on each of the dataset-specific Dice coefficients. Results of these tests were then combined across the two datasets (see Materials and Methods). This analysis demonstrated differences in neighborhood relationships between the cerebrum and cerebellum (Dataset $1, p=0.02$; Dataset2, $p=0.048$ permutation tests; meta-analysis: $\chi_{(4)}^{2}=13.59, p=0.009$ Fisher's method, $\alpha=0.05$ ), reflecting that the representations of the lip and foot show more similar levels of overlap with the hand representation in the cerebral cortex (Makin et al., 2015) relative to the cerebellum (Fig. 8C).

\section{Discussion}

Here we report large-scale remapping of body-part representations in both the cerebellar and cerebral cortices of one-handers, and provide similar preliminary results in the putamen. In all terminals, the residual arm, lips, and feet excessively activated the deprived hand region of one-handers compared to controls (Figs. $1,4,7)$. Remapping was specific to the deprived hand regions of these terminals (as reflected in our whole-brain analyses and significant group $\times$ hemisphere interactions), despite differences in the somatotopic layouts across these sensorimotor terminals (Manni and Petrosini, 2004; Mottolese et al., 2013, 2015; Makin et al., 2015). Our findings therefore challenge the view that sensorimotor remapping is restricted by the underlying somatotopy of the remapped regions (Merzenich et al., 1984; Pons et al., 1991; Merzenich and Jenkins, 1993; Faggin et al., 1997; Florence et al., 
Table 6. Meta-analysis statistics for cerebral cortex activations ${ }^{a}$

\begin{tabular}{llll}
\hline & Residual arm & Lips & Feet \\
\hline Group contrast & $\chi_{(6)}^{2}=47.73, p<0.001$ & $\chi_{(6)}^{2}=43.8, p<0.001$ & $\chi_{(6)}^{2}=37.08, p<0.001$ \\
Group $\times$ hemisphere interaction & $\chi_{(6)}^{2}=32.54, p<0.001$ & $\chi_{(6)}^{2}=28.07, p<0.001$ & $\chi_{(6)}^{2}=8.31, p=0.22$ \\
\hline
\end{tabular}

${ }^{a}$ Fisher's method statistics ( $\chi^{2}$ and $p$ value) are presented for the between-group contrasts and group $\times$ hemisphere interactions (rows) across experimental conditions (columns). All $p$ values are Bonferroni corrected ( $\alpha=0.017$ ).

Table 7. Assessment of the consistency of results across datasets using additional meta-analysis methods ${ }^{a}$

\begin{tabular}{|c|c|c|c|c|c|c|c|c|c|}
\hline \multirow[b]{2}{*}{ Brain region } & \multirow[b]{2}{*}{ Test } & \multicolumn{2}{|c|}{ Residual arm } & \multicolumn{2}{|l|}{ Lips } & \multicolumn{2}{|l|}{ Feet } & \multicolumn{2}{|c|}{ Intact hand } \\
\hline & & $P_{s}$ & $P_{z}$ & $P_{s}$ & $P_{z}$ & $P_{s}$ & $P_{z}$ & $P_{s}$ & $P_{z}$ \\
\hline \multirow[t]{2}{*}{ Cerebellum } & Group contrasts & 0.002 & 0.002 & $<0.001$ & $<0.001$ & $<0.001$ & $<0.001$ & 0.41 & 0.42 \\
\hline & Group $\times$ hemisphere interaction & $<0.001$ & $<0.001$ & $<0.001$ & $<0.001$ & $<0.001$ & 0.001 & 0.4 & 0.4 \\
\hline \multirow[t]{2}{*}{ Cerebrum } & Group contrasts & $<0.001$ & $<0.001$ & $<0.001$ & $<0.001$ & $<0.001$ & $<0.001$ & 0.13 & 0.13 \\
\hline & Group $\times$ hemisphere interaction & $<0.001$ & $<0.001$ & $<0.001$ & $<0.001$ & 0.001 & $<0.001$ & 0.14 & 0.16 \\
\hline
\end{tabular}

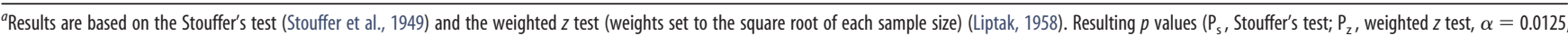
Bonferroni-corrected) are presented for the cerebrum and cerebellum hand regions (rows) and for each experimental condition (columns).

1998; Margolis et al., 2012; Striem-Amit et al., 2018), at least following congenital deprivation.

Previous studies of similar sensorimotor-deprived populations, relying on relatively small sample sizes, produced mixed evidence for the extent and drivers of remapping. Here we used a large imaging database of one-handers $(n=26)$, and demonstrated the reproducibility of our main results across independently acquired datasets, thereby establishing statistical validity (Ioannidis et al., 2014; Picciotto, 2018). Our findings therefore contribute robust evidence that remapping extends beyond the boundaries of the somatotopy, and emphasize the need to consider sensorimotor remapping following congenital malformation as a more complex phenomenon than has previously been discussed. Future large-scale studies of both functional representation and connectivity, as well as stimulation studies (e.g., transcranial magnetic stimulation) (Stoeckel et al., 2009) will be needed to fully understand the functional specificity and underlying factors that drive the reported remapping.

If remapping into the deprived hand region is not exclusively restricted to the neighboring representations, what other factors determine which representation undergoes remapping and which does not? One possibility is that remapping is shaped by altered inputs to the deprived cortex, due to compensatory behavior. We have previously characterized the behavioral repertoire of one-handers, which comprises utilization of their residual arm, lips, and feet to compensate for their hand absence (Hahamy et al., 2017). As previously reported and further extended here, the same body parts used for compensatory purposes also remap onto the deprived hand regions of both the cerebel-
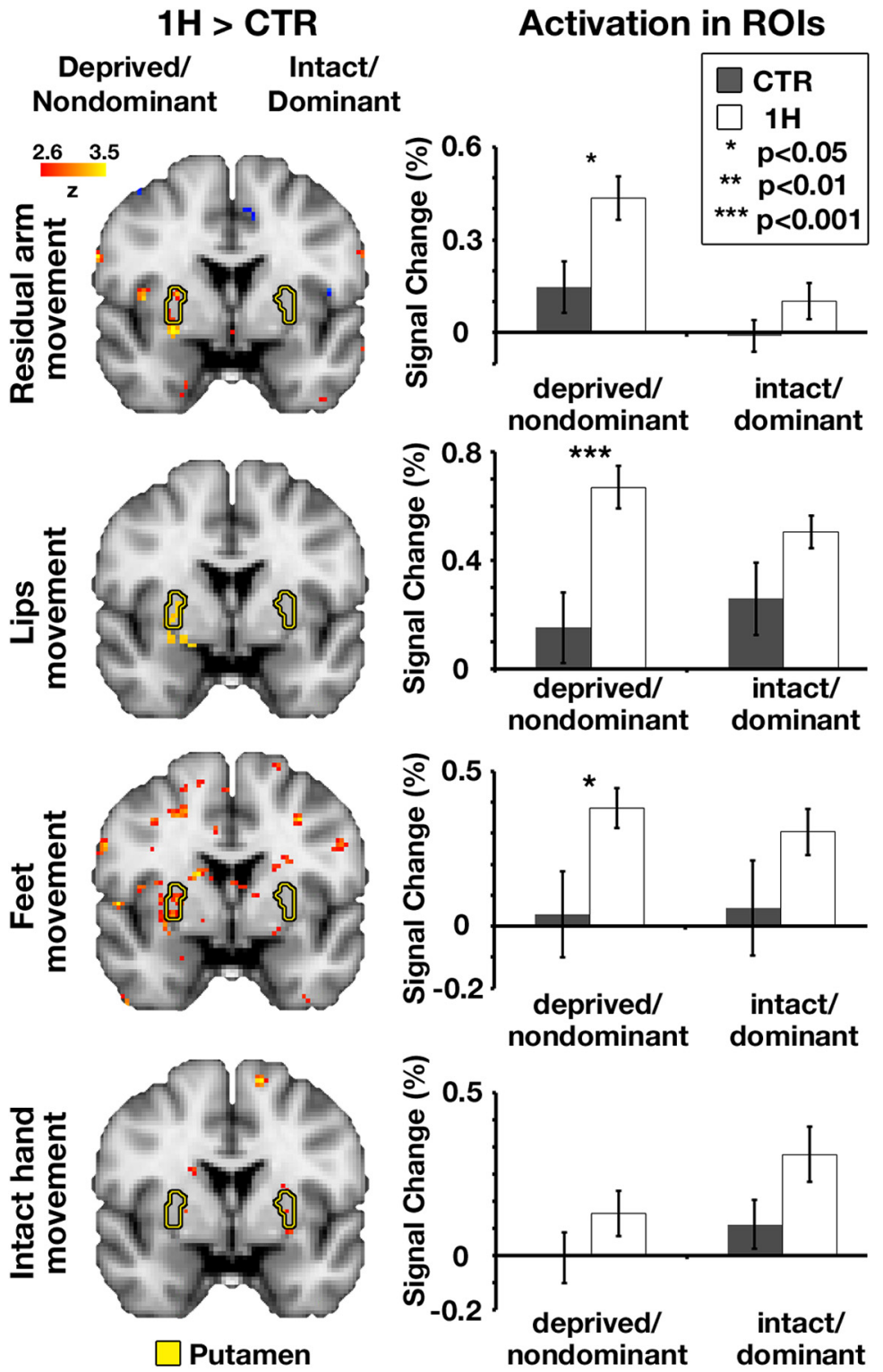

Figure 7. Multiple body parts overactivated the deprived putamen of one-handers. Left, Between-group contrast maps of Dataset2, during residual/nondominant arm (one-handers/controls), lips, feet, and intact/dominant hand movements. In each of the arm, lips, and feet (but not intact hand) conditions, one-handers showed increased activation compared with controls, centered on the deprived putamen. Yellow contours represent the bilateral putamen nuclei. Between-group contrast maps of the residual arm, feet, and intact hand conditions are presented at an uncorrected threshold of $p<0.01$. Right, Activation levels in Dataset 2 in the bilateral putamen nuclei, during each of the experimental conditions. Activation levels in the deprived putamen of one-handers (white bars) were greater than in the nondominant putamen of controls (gray bars) in all but the intact hand condition. All annotations are as in Figure 3. 
A sensorimotor cerebral cortex

sensorimotor cerebellar cortex

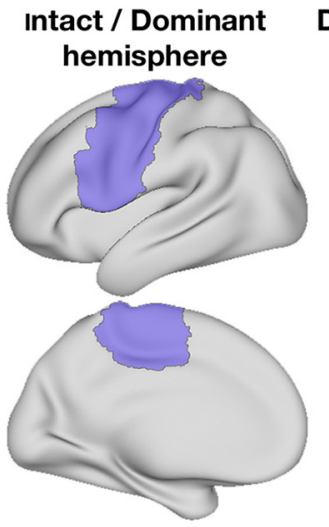

\section{Deprived / Nondominant Intact / Dominant hemisphere hemisphere}

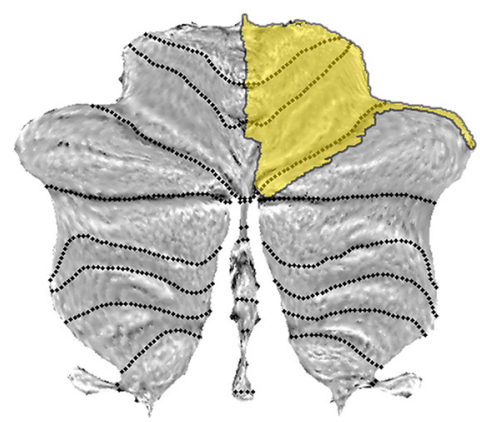

B

Dataset1
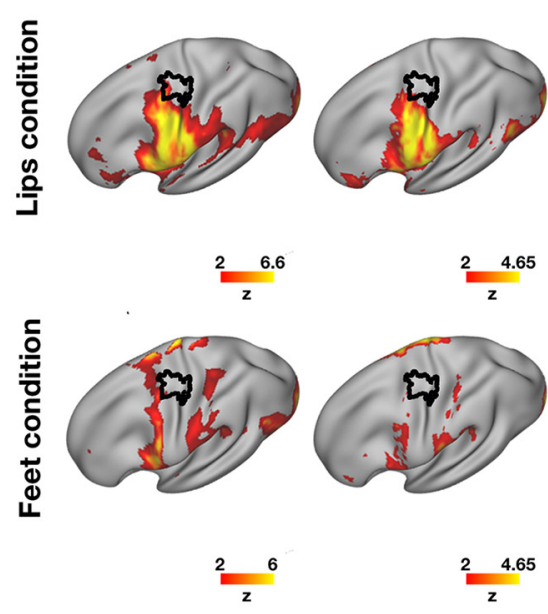

Dataset2
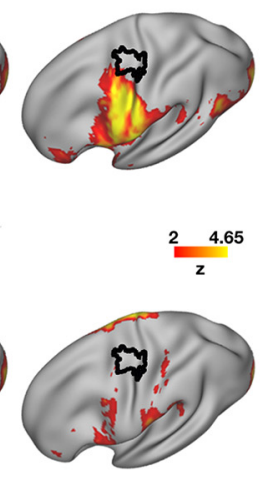

Dataset1

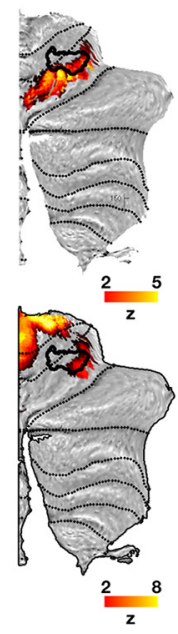

*

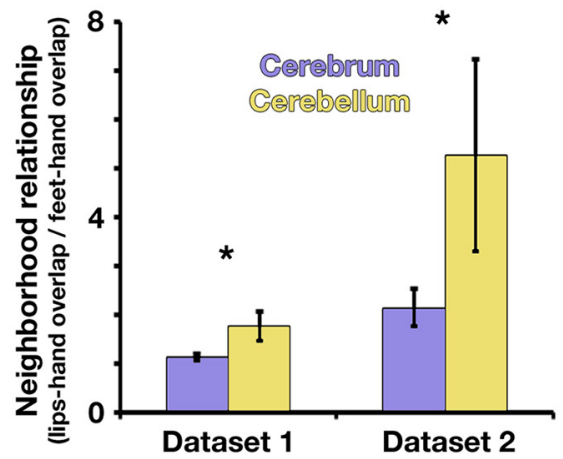

Figure 8. Different overlap relationships of body-part activations between the cerebrum and cerebellum. $\boldsymbol{A}$, Sensorimotor masks used to estimate overlap relationships between body-part representations in the intact/dominant cerebral hemisphere (left, marked in purple over an inflated cortical surface) and intact/dominant cerebellar hemisphere (right, marked in yellow over a flattened cerebellar surface). The "intact/dominant" cerebral hemisphere is contralateral to the intact/dominant hand (onehanders/controls, respectively) and the "intact/dominant" cerebellar hemisphere is ipsilateral to the intact/dominant hand. $\boldsymbol{B}$, Averaged maps across all participants of each dataset (columns) in the lips (top row) and feet (bottom row) condition, projected onto surfaces of the intact/dominant cerebral (left) and cerebellar (right) hemispheres. Independent ROls of the intact/dominant hand are depicted in black contours on these same surfaces. These ROls are presented for illustration purposes only, and were not used in our statistical analysis of neighborhood relationships, which does not rely on ROls (see Materials and Methods). C, Overlap between (1) hand and lips activations and (2) hand and feet activations were estimated for each participant using the Dice coefficient (see Materials and Methods). The relationship between these overlapping activations was calculated as the ratio between lips-hand overlap and feet-hand overlap ( $y$ axis), which was calculated separately for the cerebrum (purple bars) and cerebellum (yellow bars) within each separate dataset. As evident in both datasets, the ratios of overlap between hand-lips and hand activations are larger in the cerebellum compared with the cerebral cortex (cerebellar ratios $>$ cerebral ratios), demonstrating different somatotopic layout of body part representations between the cerebral and cerebellar cortices. ${ }^{*} p<0.05$. lum and cerebrum. Furthermore, the intact hand, which is not overused for compensatory purposes in one-handers (Makin et al., 2013b; Philip and Frey, 2014; Hahamy et al., 2017), does not show remapping onto either the cerebellar or cerebral deprived hand regions. However, we cannot exclude the possibility that remapping in the deprived hand region of one-handers is restricted to body-part representations within the deprived hemisphere. This is because, in the current experimental design, the intact hand is the only body part contralateral to the deprived cerebral hemisphere/ ipsilateral to the deprived cerebellar hemisphere.

It is important to mention that so far we have been unable to identify a correlation between individuals' idiosyncratic compensatory strategies and brain remapping. Moreover, other studies reported large-scale remapping dissociated from compensatory behavior in congenital or juvenile bilateral hand loss (Yu et al., 2014; Striem-Amit et al., 2018). For example, Striem-Amit et al. (2018) recently demonstrated that body-part representations neighboring the deprived hand region can show remapping, even if these body parts are not prominently used for compensatory purposes. These discrepancies across studies could be attributed to the fact that compensatory daily behavior is difficult to quantify comprehensively and reliably. Alternatively, it could be speculated that the development of one-handers' intact hand grants the deprived hand region some sensorimotor scaffolding relating to hand functionality (e.g., via interhemispheric functional connectivity) (Hahamy et al., 2017). This, in turn, may guide remapping in onehanders based on behavioral criteria (e.g., relevance for supporting the intact hand), which will not be available or functionally relevant in cases of bilateral hand malformation. We also cannot exclude the possibility that behavior and brain remapping may not be directly related. For example, the observed remapping in one-handers may merely reflect weak normal inputs from different body parts to the hand region, which are typically suppressed. In the absence of a hand, these inputs may simply be unmasked, and not necessarily causally support compensatory behavior (Makin and Bensmaia, 2017; for further discussion see Krakauer and Carmichael, 2017). Taken together, further research is needed to validate the causal origins and consequences of behavior on the large-scale remapping reported here. 
Similar controversy regarding the role of somatotopic boundaries in shaping remapping also exists in amputation research. In adult amputees, remapping is commonly attributed to residual arm representation (Kew et al., 1994; Irlbacher et al., 2002; Raffin et al., 2016; but see Gagné et al., 2011; Makin et al., 2013b) and mouth representation (Flor et al., 1995; Elbert et al., 1997; Karl et al., 2001; Lotze et al., 2001; MacIver et al., 2008; Foell et al., 2014; but see Makin et al., 2013a, 2015; Raffin et al., 2016), both thought to neighbor the hand region (but see Zeharia et al., 2015; Roux et al., 2018; as well as hand and lip regions in Figure 4). More recent findings reveal remapping of the intact hand representation into the deprived cortex (Bogdanov et al., 2012; Makin et al., 2013b; Philip and Frey, 2014). These more recent findings have ascribed remapping to the compensatory use of amputees' intact hand. Thus, findings across varied sensorimotor-deprived populations raise the possibility that body-part representations that have little overlap, if any, with the hand region (lips and feet in one-handers, feet in individuals with bilateral congenital limb loss or childhood amputation, and intact hand in amputated adults) can remap onto the deprived hand region.

Although we discuss commonalities in remapping across the life span, this is not to imply that remapping bears the same mechanistic and functional meaning when occurring at different life stages. Hand function begins to form in utero (Zoia et al., 2007) and continues to develop into late childhood (KuhtzBuschbeck et al., 1998). As such, congenital hand malformation offers multiple opportunities for functional remapping during development. Indeed, vast research on visual and auditory deprivations introduced the notion of the critical period, an early period of life in which sensory experience may have greater impact on brain remapping and consequent behavior, compared with later periods (Voss, 2013; for review, see Kral, 2013). In contrast, classical research of sensorimotor deprivation documented extensive remapping in adults (Pons et al., 1991; Florence et al., 1998). Although the extent and functional significance of remapping in later life are still debated (Collignon et al., 2013; Bedny, 2017; Makin and Bensmaia, 2017; Singh et al., 2018), it is worth noting that, compared with congenital blindness and deafness research, sensorimotor deprivation is confined to the sensorimotor network, and is thus smaller in scale. Therefore, amputation-related deprivation might provide more opportunities/restrictions for remapping across the life span, meaning that sensorimotor remapping may still be feasible in adulthood (Dempsey-Jones et al., 2019).

Finally, the remapping reported here may indeed be constrained by proximity between body-part representations, not in the cerebellum/cerebrum, but rather in subcortical sensorimotor terminals. While it has originally been suggested that sensorimotor remapping occurs at the level of the cerebral cortex (Pons et al., 1991; Florence et al., 1998), recent studies in monkeys emphasize the role of subcortical structures, such as the brainstem, in which the layout of somatotopic representations differs from that of the cerebral cortex (Jain et al., 2000; Kambi et al., 2014; Chand and Jain, 2015; Liao et al., 2016). For example, Kambi et al. (2014) demonstrated that facial remapping in the deprived somatosensory hand region of spinal-cord-injured monkeys is abolished upon inactivation of the deprived cuneate nucleus. The fact that the cuneate nucleus does not normally receive inputs from the face suggests that remapping seen in the cerebral cortex is likely driven by reorganization at the level of the brainstem (for a related example of remapping in the motor cortex, see also Herbert et al., 2015). It is therefore plausible that the remapping we observed in one-handers may also be initiated in upstream sensorimotor terminal.

Indeed, our data provide initial evidence for remapping in one-handers' putamen, which mirrors the remapping patterns of the cerebral and cerebellar cortices. Interestingly, representations of the hand, lip, and foot, which are distant in the cerebral/cerebellar cortices, neighbor each other in the putamen of the human basal ganglia (Gerardin et al., 2003; Staempfli et al., 2008). These neighboring representations may thus more easily remap onto the deprived hand region. Consequently, projections from the putamen's hand representation to its cerebral/cerebellar counterparts would appear as remapping that is independent of the cerebral/cerebellar somatotopic layouts. This hypothesis is consistent with anatomical evidence for a closed-loop, reciprocal circuit between primary sensorimotor cortex, cerebellum, and basal ganglia, such that each terminal projects to and receives inputs from each other terminal, with varying somatotopic layouts within each terminal (Nambu, 2011; Dum et al., 2014; Zeharia et al., 2015). As our findings reveal remapping in all three of these interconnected terminals, it is plausible that the documented remapping is initiated by the basal ganglia, or further upstream. However, the results we reported in the putamen could only be observed in one dataset, and lacked the spatial resolution to accurately allocate the position of the putamen hand region. These results therefore await further confirmation with more specialized data collection tools. In addition, since our results demonstrate remapping in both primary somatosensory and motor cortices (Fig. 4), which have differing upstream hierarchies, the role of subcortical structures in driving cortical remapping requires further research. Nevertheless, it is tempting to speculate that the upstream sensorimotor terminal at which remapping may be initiated would contain a somatotopic layout specifically suitable for supporting the emerging repertoire of compensatory behaviors.

\section{References}

Andoh J, Milde C, Tsao JW, Flor H (2018) Cortical plasticity as a basis of phantom limb pain: fact or fiction? Neuroscience 387:85-91.

Bedny M (2017) Evidence from blindness for a cognitively pluripotent cortex. Trends Cogn Sci 21:637-648.

Bogdanov S, Smith J, Frey SH (2012) Former hand territory activity increases after amputation during intact hand movements, but is unaffected by illusory visual feedback. Neurorehabil Neural Repair 26:604-615.

Buckner RL, Krienen FM, Castellanos A, Diaz JC, Yeo BT (2011) The organization of the human cerebellum estimated by intrinsic functional connectivity. J Neurophysiol 106:2322-2345.

Carp J (2012) On the plurality of (methodological) worlds: estimating the analytic flexibility of FMRI experiments. Front Neurosci 6:149.

Catani M (2017) A little man of some importance. Brain 140:3055-3061.

Chand P, Jain N (2015) Intracortical and thalamocortical connections of the hand and face representations in somatosensory area $3 \mathrm{~b}$ of macaque monkeys and effects of chronic spinal cord injuries. J Neurosci 35: 13475-13486.

Collignon O, Dormal G, Albouy G, Vandewalle G, Voss P, Phillips C, Lepore F (2013) Impact of blindness onset on the functional organization and the connectivity of the occipital cortex. Brain 136:2769-2783.

Dempsey-Jones H, Themistocleous AC, Carone D, Ng TW, Harrar V, Makin TR (2019) Blocking tactile input to one finger using anaesthetic enhances touch perception and learning in other fingers. J Exp Psychol Gen 148:713-727.

Dice LR (1945) Measures of the amount of ecologic association between species. Ecology 26:297-302.

Diedrichsen J, Zotow E (2015) Surface-based display of volume-averaged cerebellar imaging data. PLoS One 10:e133402.

Dum RP, Bostan AC, Strick PL (2014) 36 Basal ganglia and cerebellar circuits with the cerebral cortex. In: The cognitive neurosciences: Chap 36, pp 419-434. Massachusetts: MIT. 
Elbert T, Sterr A, Flor H, Rockstroh B, Knecht S, Pantev C, Wienbruch C, Taub E (1997) Input-increase and input-decrease types of cortical reorganization after upper extremity amputation in humans. Exp Brain Res 117:161-164.

Faggin BM, Nguyen KT, Nicolelis MA (1997) Immediate and simultaneous sensory reorganization at cortical and subcortical levels of the somatosensory system. Proc Natl Acad Sci U S A 94:9428-9433.

Fisher R (1925) Statistical methods for research workers. Guildford, UK: Genesis.

Fisher R (1948) Questions and answers, 14. Am Statistician 2:30-31.

Flor H, Elbert T, Knecht S, Wienbruch C, Pantev C, Birbaumer N, Larbig W, Taub E (1995) Phantom-limb pain as a perceptual correlate of cortical reorganization following arm amputation. Nature 375:482-484.

Florence SL, Taub HB, Kaas JH (1998) Large-scale sprouting of cortical connections after peripheral injury in adult macaque monkeys. Science 282:1117-1121.

Foell J, Bekrater-Bodmann R, Diers M, Flor H (2014) Mirror therapy for phantom limb pain: brain changes and the role of body representation. Eur J Pain 18:729-739.

Friston K (2012) Ten ironic rules for non-statistical reviewers. Neuroimage 61:1300-1310.

Gagné M, Hétu S, Reilly KT, Mercier C (2011) The map is not the territory: motor system reorganization in upper limb amputees. Hum Brain Mapp 32:509-519.

Gerardin E, Lehéricy S, Pochon JB, Tézenas du Montcel S, Mangin JF, Poupon F, Agid Y, Le Bihan D, Marsault C (2003) Foot, hand, face and eye representation in the human striatum. Cereb Cortex 13:162-169.

Greve DN, Fischl B (2009) Accurate and robust brain image alignment using boundary-based registration. Neuroimage 48:63-72.

Grodd W, Hülsmann E, Lotze M, Wildgruber D, Erb M (2001) Sensorimotor mapping of the human cerebellum: fMRI evidence of somatotopic organization. Hum Brain Mapp 13:55-73.

Haak KV, Marquand AF, Beckmann CF (2018) Connectopic mapping with resting-state fMRI. Neuroimage 170:83-94.

Hahamy A, Behrmann M, Malach R (2015a) The idiosyncratic brain: distortion of spontaneous connectivity patterns in autism spectrum disorder. Nat Neurosci 18:302-309.

Hahamy A, Sotiropoulos SN, Henderson Slater D, Malach R, Johansen-Berg H, Makin TR (2015b) Normalisation of brain connectivity through compensatory behaviour, despite congenital hand absence. eLife 4:04605.

Hahamy A, Macdonald SN, van den Heiligenberg F, Kieliba P, Emir U, Malach R, Johansen-Berg H, Brugger P, Culham JC, Makin TR (2017) Representation of multiple body parts in the missing-hand territory of congenital one-handers. Curr Biol 27:1350-1355.

Herbert WJ, Powell K, Buford JA (2015) Evidence for a role of the reticulospinal system in recovery of skilled reaching after cortical stroke: initial results from a model of ischemic cortical injury. Exp Brain Res 233:32313251.

Holmes AP, Blair RC, Watson JD, Ford I (1996) Nonparametric analysis of statistic images from functional mapping experiments. J Cereb Blood Flow Metab 16:7-22.

Ioannidis JP, Munafò MR, Fusar-Poli P, Nosek BA, David SP (2014) Publication and other reporting biases in cognitive sciences: detection, prevalence, and prevention. Trends Cogn Sci 18:235-241.

Irlbacher K, Meyer BU, Voss M, Brandt SA, Röricht S (2002) Spatial reorganization of cortical motor output maps of stump muscles in human upper-limb amputees. Neurosci Lett 321:129-132.

Jain N, Florence SL, Qi HX, Kaas JH (2000) Growth of new brainstem connections in adult monkeys with massive sensory loss. Proc Natl Acad Sci U S A 97:5546-5550.

Jain N, Qi HX, Collins CE, Kaas JH (2008) Large-scale reorganization in the somatosensory cortex and thalamus after sensory loss in macaque monkeys. J Neurosci 28:11042-11060.

Jang JH, Jung WH, Choi JS, Choi CH, Kang DH, Shin NY, Hong KS, Kwon JS (2011) Reduced prefrontal functional connectivity in the default mode network is related to greater psychopathology in subjects with high genetic loading for schizophrenia. Schizophr Res 127:58-65.

Jenkinson M, Bannister P, Brady M, Smith S (2002) Improved optimization for the robust and accurate linear registration and motion correction of brain images. Neuroimage 17:825-841.

Kaas JH, Qi HX, Burish MJ, Gharbawie OA, Onifer SM, Massey JM (2008) Cortical and subcortical plasticity in the brains of humans, primates, and rats after damage to sensory afferents in the dorsal columns of the spinal cord. Exp Neurol 209:407-416.

Kambi N, Halder P, Rajan R, Arora V, Chand P, Arora M, Jain N (2014) Large-scale reorganization of the somatosensory cortex following spinal cord injuries is due to brainstem plasticity. Nat Commun 5:3602.

Karl A, Birbaumer N, Lutzenberger W, Cohen LG, Flor H (2001) Reorganization of motor and somatosensory cortex in upper extremity amputees with phantom limb pain. J Neurosci 21:3609-3618.

Kew JJ, Ridding MC, Rothwell JC, Passingham RE, Leigh PN, Sooriakumaran S, Frackowiak RS, Brooks DJ (1994) Reorganization of cortical blood flow and transcranial magnetic stimulation maps in human subjects after upper limb amputation. J Neurophysiol 72:2517-2524.

Kikkert S, Kolasinski J, Jbabdi S, Tracey I, Beckmann CF, Johansen-Berg H, Makin TR (2016) Revealing the neural fingerprints of a missing hand. Elife 5:e15292.

Krakauer JW, Carmichael ST (2017) Broken movement: the neurobiology of motor recovery after stroke. Cambridge, MA: Massachusetts Institute of Technology.

Kral A (2013) Auditory critical periods: a review from system's perspective. Neuroscience 247:117-133.

Kuhtz-Buschbeck JP, Stolze H, Jöhnk K, Boczek-Funcke A, Illert M (1998) Development of prehension movements in children: a kinematic study. Exp Brain Res 122:424-432.

Liao CC, Reed JL, Kaas JH, Qi HX (2016) Intracortical connections are altered after long-standing deprivation of dorsal column inputs in the hand region of area 3b in squirrel monkeys. J Comp Neurol 524:1494-1526.

Liptak T (1958) On the combination of independent tests. Magyar Tud Akad Mat Kutato Int Kozl 3:171-197.

Lotze M, Flor H, Grodd W, Larbig W, Birbaumer N (2001) Phantom movements and pain: an fMRI study in upper limb amputees. Brain 124:2268 2277.

MacIver K, Lloyd DM, Kelly S, Roberts N, Nurmikko T (2008) Phantom limb pain, cortical reorganization and the therapeutic effect of mental imagery. Brain 131:2181-2191.

Makin TR, Bensmaia SJ (2017) Stability of sensory topographies in adult cortex. Trends Cogn Sci 21:195-204.

Makin TR, Scholz J, Filippini N, Henderson Slater D, Tracey I, Johansen-Berg $\mathrm{H}$ (2013a) Phantom pain is associated with preserved structure and function in the former hand area. Nat Commun 4:1570.

Makin TR, Cramer AO, Scholz J, Hahamy A, Henderson Slater D, Tracey I, Johansen-Berg H (2013b) Deprivation-related and use-dependent plasticity go hand in hand. Elife 2:e01273.

Makin TR, Scholz J, Henderson Slater D, Johansen-Berg H, Tracey I (2015) Reassessing cortical reorganization in the primary sensorimotor cortex following arm amputation. Brain 138:2140-2146.

Manni E, Petrosini L (2004) A century of cerebellar somatotopy: a debated representation. Nat Rev Neurosci 5:241-249.

Margolis DJ, Lütcke H, Schulz K, Haiss F, Weber B, Kügler S, Hasan MT, Helmchen F (2012) Reorganization of cortical population activity imaged throughout long-term sensory deprivation. Nat Neurosci 15:15391546.

Masyn K, Nathan P, Little T (2013) The Oxford handbook of quantitative methods, Vol 2: Statistical analysis. Oxford: Oxford Library of Psychology.

Merzenich MM, Jenkins WM (1993) Reorganization of cortical representations of the hand following alterations of skin inputs induced by nerve injury, skin island transfers, and experience. J Hand Ther 6:89-104.

Merzenich MM, Nelson RJ, Stryker MP, Cynader MS, Schoppmann A, Zook JM (1984) Somatosensory cortical map changes following digit amputation in adult monkeys. J Comp Neurol 224:591-605.

Mottolese C, Richard N, Harquel S, Szathmari A, Sirigu A, Desmurget M (2013) Mapping motor representations in the human cerebellum. Brain 136:330-342

Mottolese C, Szathmari A, Beuriat PA, Sirigu A, Desmurget M (2015) Sensorimotor mapping of the human cerebellum during pineal region surgery. Neurochirurgie 61:101-105.

Nambu A (2011) Somatotopic organization of the primate basal ganglia. Front Neuroanat 5:26.

Nichols TE, Holmes AP (2002) Nonparametric permutation tests for functional neuroimaging: a primer with examples. Hum Brain Mapp 15:1-25.

Penfield W, Boldrey E (1937) Somatic motor and sensory representation in 
the cerebral cortex of man as studied by electrical stimulation. Brain 60:389-443.

Penfield W, Rasmussen T (1950) The cerebral cortex of man: a clinical study of localization of function. Oxford: Macmillan.

Philip BA, Frey SH (2014) Compensatory changes accompanying chronic forced use of the nondominant hand by unilateral amputees. J Neurosci 34:3622-3631.

Picciotto M (2018) Analytical transparency and reproducibility in human neuroimaging studies. J Neurosci 38:3375-3376.

Pons TP, Garraghty PE, Ommaya AK, Kaas JH, Taub E, Mishkin M (1991) Massive cortical reorganization after sensory deafferentation in adult macaques. Science 252:1857-1860.

Raffin E, Richard N, Giraux P, Reilly KT (2016) Primary motor cortex changes after amputation correlate with phantom limb pain and the ability to move the phantom limb. Neuroimage 130:134-144.

Roux FE, Djidjeli I, Durand JB (2018) Functional architecture of the somatosensory homunculus detected by electrostimulation. J Physiol 596:941-956.

Singh AK, Phillips F, Merabet LB, Sinha P (2018) Why does the cortex reorganize after sensory loss? Trends Cogn Sci 22:569-582.

Smith SM (2002) Fast robust automated brain extraction. Hum Brain Mapp 17:143-155.

Staempfli P, Reischauer C, Jaermann T, Valavanis A, Kollias S, Boesiger P (2008) Combining fMRI and DTI: a framework for exploring the limits of fMRI-guided DTI fiber tracking and for verifying DTI-based fiber tractography results. Neuroimage 39:119-126.
Stoeckel MC, Seitz RJ, Buetefisch CM (2009) Congenitally altered motor experience alters somatotopic organization of human primary motor cortex. Proc Natl Acad Sci U S A 106:2395-2400.

Stouffer SA, Suchman EA, DeVinney LC, Star SA, Williams Jr RM (1949) The American soldier. Princeton, NJ: Princeton UP.

Striem-Amit E, Vannuscorps G, Caramazza A (2018) Plasticity based on compensatory effector use in the association but not primary sensorimotor cortex of people born without hands. Proc Natl Acad Sci U S A 115:7801-7806.

Voss P (2013) Sensitive and critical periods in visual sensory deprivation. Front Psychol 4:664.

Wiestler T, McGonigle DJ, Diedrichsen J (2011) Integration of sensory and motor representations of single fingers in the human cerebellum. J Neurophysiol 105:3042-3053.

Yu XJ, He HJ, Zhang QW, Zhao F, Zee CS, Zhang SZ, Gong XY (2014) Somatotopic reorganization of hand representation in bilateral arm amputees with or without special foot movement skill. Brain Res 1546:9-17.

Zeharia N, Hertz U, Flash T, Amedi A (2015) New whole-body sensorymotor gradients revealed using phase-locked analysis and verified using multivoxel pattern analysis and functional connectivity. J Neurosci 35:2845-2859.

Zoia S, Blason L, D’Ottavio G, Bulgheroni M, Pezzetta E, Scabar A, Castiello U (2007) Evidence of early development of action planning in the human foetus: a kinematic study. Exp Brain Res 176:217-226. 